ARTICLE

\title{
Molecular structure and interactions within amyloid-like fibrils formed by a low-complexity protein sequence from FUS
}

\author{
Myungwoon Lee ${ }^{1}$, Ujjayini Ghosh ${ }^{1}$, Kent R. Thurber ${ }^{1}$, Masato Kato ${ }^{2,3}$ \& Robert Tycko ${ }^{1 凶}$
}

Protein domains without the usual distribution of amino acids, called low complexity (LC) domains, can be prone to self-assembly into amyloid-like fibrils. Self-assembly of LC domains that are nearly devoid of hydrophobic residues, such as the 214-residue LC domain of the RNA-binding protein FUS, is particularly intriguing from the biophysical perspective and is biomedically relevant due to its occurrence within neurons in amyotrophic lateral sclerosis, frontotemporal dementia, and other neurodegenerative diseases. We report a high-resolution molecular structural model for fibrils formed by the C-terminal half of the FUS LC domain (FUS-LC-C, residues $111-214$ ), based on a density map with $2.62 \AA$ resolution from cryoelectron microscopy (cryo-EM). In the FUS-LC-C fibril core, residues 112-150 adopt U-shaped conformations and form two subunits with in-register, parallel cross $-\beta$ structures, arranged with quasi-2 $2_{1}$ symmetry. All-atom molecular dynamics simulations indicate that the FUS-LCC fibril core is stabilized by a plethora of hydrogen bonds involving sidechains of Gln, Asn, Ser, and Tyr residues, both along and transverse to the fibril growth direction, including diverse sidechain-to-backbone, sidechain-to-sidechain, and sidechain-to-water interactions. Nuclear magnetic resonance measurements additionally show that portions of disordered residues 151-214 remain highly dynamic in FUS-LC-C fibrils and that fibrils formed by the Nterminal half of the FUS LC domain (FUS-LC-N, residues 2-108) have the same core structure as fibrils formed by the full-length LC domain. These results contribute to our understanding of the molecular structural basis for amyloid formation by FUS and by LC domains in general.

\footnotetext{
${ }^{1}$ Laboratory of Chemical Physics, National Institute of Diabetes and Digestive and Kidney Diseases, National Institutes of Health, Bethesda, Maryland $20892-$ 0520, USA. ${ }^{2}$ Department of Biochemistry, University of Texas Southwestern Medical Center, 5323 Harry Hines Boulevard, Dallas, Texas 75390-9152, USA. ${ }^{3}$ Institute for Quantum Life Science, National Institutes for Quantum and Radiological Science and Technology, Chiba 263-8555, Japan.

凶email: robertty@mail.nih.gov
} 
C urrent interest in self-assembly processes involving proteins with domains of low sequence complexity (LC domains) arises from observations that liquid-liquid phase separation (LLPS) by such proteins within eukaryotic cells has a variety of biological functions ${ }^{1,2}$, that LLPS by proteins with LC domains can lead to hydrogel formation in which their LC domains form the structurally ordered cores of amyloid-like fibrils $^{3,4}$, and that amyloid aggregation by proteins with LC domains is associated with neurodegenerative diseases ${ }^{5-7}$.

FUS (FUsed in Sarcoma) is a $54 \mathrm{kDa}$ RNA-binding protein with multiple functions, including roles in the regulation of transcription through interactions with the C-terminal domain of RNA polymerase $\mathrm{II}^{8,9}$, in RNA splicing through multimeric binding to long introns ${ }^{10,11}$, in DNA damage repair ${ }^{12,13}$, in cellular responses to stress ${ }^{14,15}$, and in mRNA transport and subcellular localization of translation ${ }^{16}$. The C-terminal half of FUS contains RNA recognition, zinc finger, Arg-Gly-Gly, and nuclear localization signal (NLS) domains. The N-terminal half is an LC domain comprised primarily of Ser, Gly, Gln, Tyr, Thr, and Pro residues, and with no Leu, Ile, Val, or Phe residues. Although the LC domain of FUS is largely unstructured as a monomer in solution ${ }^{17}$, the LC domain is essential for normal FUS functions, including shuttling between the nucleus and cytoplasm ${ }^{18}$, DNA damage repair ${ }^{12}$, and transcription regulation ${ }^{19,20}$.

In vitro, solutions of either full-length FUS or its LC domain alone undergo LLPS in a temperature-dependent and concentration-dependent manner. Phase-separated high-concentration droplets of full-length FUS or its LC domain typically evolve over time to a hydrogel state, consisting of highly entangled, amyloid-like fibrils ${ }^{3}$, or to precipitated fibril bundles ${ }^{21,22}$. Thus, liquid-like FUS droplets represent a metastable condition ${ }^{15}$, with the thermodynamic end state being FUS fibrils.

LC-domain-dependent intracellular aggregation of FUS ${ }^{23,24}$ is also implicated in neurodegenerative diseases, especially familial amyotrophic lateral sclerosis and frontotemporal dementia, with disease-associated mutations occurring in both the NLS and the LC domains ${ }^{25-27}$. These mutations are believed to stabilize the fibrillar state of FUS ${ }^{28}$ and promote its cytoplasmic localization ${ }^{24}$, leading to either loss-of-normal-function $8,10,23$ or gain-of-toxicfunction $11,27,29$ mechanisms for neurotoxicity.

Motivated by these considerations, our laboratory recently used solid-state nuclear magnetic resonance (ssNMR) measurements to determine the molecular structure of amyloid-like fibrils formed by the full-length LC domain of FUS (FUS-LC, residues 2-214). Unexpectedly, we found that a specific 57-residue segment in the N-terminal half of FUS-LC (underlined in Fig. 1a) forms the structurally ordered cross- $\beta$-fibril core, whereas the Cterminal half of FUS-LC remains dynamically disordered $21,30,31$. Eight different algorithms for predicting amyloid-forming segments from protein sequences failed to reproduce the experimental result ${ }^{21}$, highlighting the qualitative difference between FUS-LC and amyloid-forming polypeptides such as amyloid- $\beta, \alpha-$ synuclein, and tau, whose self-assembly is driven chiefly by hydrophobic interactions.

Here we report studies of the C-terminal half of FUS-LC (FUSLC-C, residues 111-214). Although this segment is dynamically disordered in fibrils formed by full-length FUS-LC, we find that FUS-LC-C by itself readily forms highly ordered fibrils, suitable for molecular structure determination by cryogenic electron microscopy (cryo-EM). The cryo-EM density map (2.62 Å resolution) and the resulting molecular model ${ }^{32}$ show that the FUSLC-C fibril core is qualitatively different from the core of fulllength FUS-LC fibrils, in terms of both molecular conformation and overall symmetry. All-atom molecular dynamics (MD) simulations based on the cryo-EM structure provide insights into the properties of internal water and the nature of sidechain-sidechain and sidechain-backbone interactions that contribute to FUS-LC-C fibril stability.

Although we do not claim that the FUS-LC-C fibril structure discussed below has direct relevance to pathogenesis or biological function, this work provides new information about the diversity of structural motifs that can exist within LC protein fibrils, their relation to motifs in fibrils formed by other types of sequences, and the diversity of intermolecular and inter-residue interactions that can stabilize amyloid fibril structures. The majority of disease-associated mutations in the LC domain of FUS do occur after residue $111^{27}$.

\section{Results}

Both the N-terminal half and the C-terminal half FUS-LC form amyloid-like fibrils. In fibrils formed by full-length FUS-LC with the sequence in Fig. 1a, residues 39-95 form the structurally ordered cross- $\beta$ core, whereas the C-terminal half of the sequence is mainly dynamically disordered ${ }^{21,30}$. However, when FUS-LC is truncated to residues 111-214, the resulting FUS-LC-C construct readily forms amyloid-like fibrils under common solution conditions $(40 \mu \mathrm{M}$ protein concentration, $20 \mathrm{mM}$ Tris- $\mathrm{HCl}$ buffer $\mathrm{pH}$ $7.4,24^{\circ} \mathrm{C}$ ). In negative-stain transmission electron microscope (TEM) images, FUS-LC-C fibrils have a pronounced apparent width modulation with a period of $44 \pm 4 \mathrm{~nm}$ (Fig. 1b). When FUS-LC is truncated to residues $2-108$, the resulting N-terminal construct (FUS-LC-N) also forms fibrils, as expected (Fig. 1c).

Two-dimensional (2D) ${ }^{15} \mathrm{~N}-{ }^{13} \mathrm{C}$ ssNMR spectra of FUS-LC-C fibrils show sharp cross-peak signals, with full-width at halfmaximum linewidths of 1.2 and 0.6 p.p.m. in ${ }^{15} \mathrm{~N}$ and ${ }^{13} \mathrm{C}$ dimensions, respectively, consistent with a well-ordered structure. However, the extreme LC character of the FUS-LC-C sequence (34.6\% Gly, 22.1\% Ser, 19.2\% Gln, 10.6\% Tyr, 12.5\% other) leads to 2D spectra with clusters of overlapping cross-peaks (Fig. 1d and Supplementary Fig. 1a). The 2D spectra of FUS-LC-N fibrils also show overlapping cross-peaks, although with more crosspeaks that are fully or partially resolved. Cross-peak positions in 2D spectra of FUS-LC-N fibrils are in good agreement with crosspeak positions in 2D spectra of full-length FUS-LC fibrils (Fig. 1e and Supplementary Fig. 1b). Specifically, 35 resolved or partially resolved cross-peaks in the $2 \mathrm{D}{ }^{15} \mathrm{~N}-{ }^{13} \mathrm{C}$ spectrum of FUS-LC-N fibrils have chemical shift values that agree to within the ssNMR linewidths with those of cross-peaks in the corresponding spectrum of FUS-LC fibrils (Fig. 1e). More than 50 resolved or partially resolved cross-peaks in the $2 \mathrm{D}{ }^{13} \mathrm{C}-{ }^{13} \mathrm{C}$ spectrum of FUS-LC-N fibrils have chemical shift values that agree to within the ssNMR linewidths with those of cross-peaks in the corresponding spectrum of FUS-LC fibrils (Supplementary Fig. S1b). Thus, the molecular structure of the FUS-LC-N fibril core is very similar to the core structure in full-length FUS-LC fibrils determined earlier ${ }^{21}$.

Measurements of the mass-per-length (MPL) of FUS-LC-C fibrils by dark-field TEM $^{33}$ yielded a value of $41.8 \pm 1.0 \mathrm{kDa} / \mathrm{nm}$ (Supplementary Fig. 2a-c). Given that the molecular weight of a FUS-LC-C monomer is $10.02 \mathrm{kDa}$ and the spacing between $\beta$ strands in a $\beta$-sheet is $0.47-0.48 \mathrm{~nm}$, the MPL value for a single cross- $\beta$ subunit in FUS-LC-C fibrils is expected to be about 21 $\mathrm{kDa} / \mathrm{nm}$, assuming a cross- $\beta$ motif with one monomer per $\beta$ sheet spacing. The experimental MPL value therefore suggests a structure containing two cross- $\beta$ subunits. In contrast, full-length FUS-LC fibrils contain a single cross- $\beta$ unit $^{21}$.

Cryo-EM reveals a two-fold symmetric core in FUS-LC-C fibrils. To determine the molecular structure of FUS-LC-C fibrils, we used cryo-EM and helical reconstruction in RELION 3.0 34,35 , with software modifications to include orientational correlations 

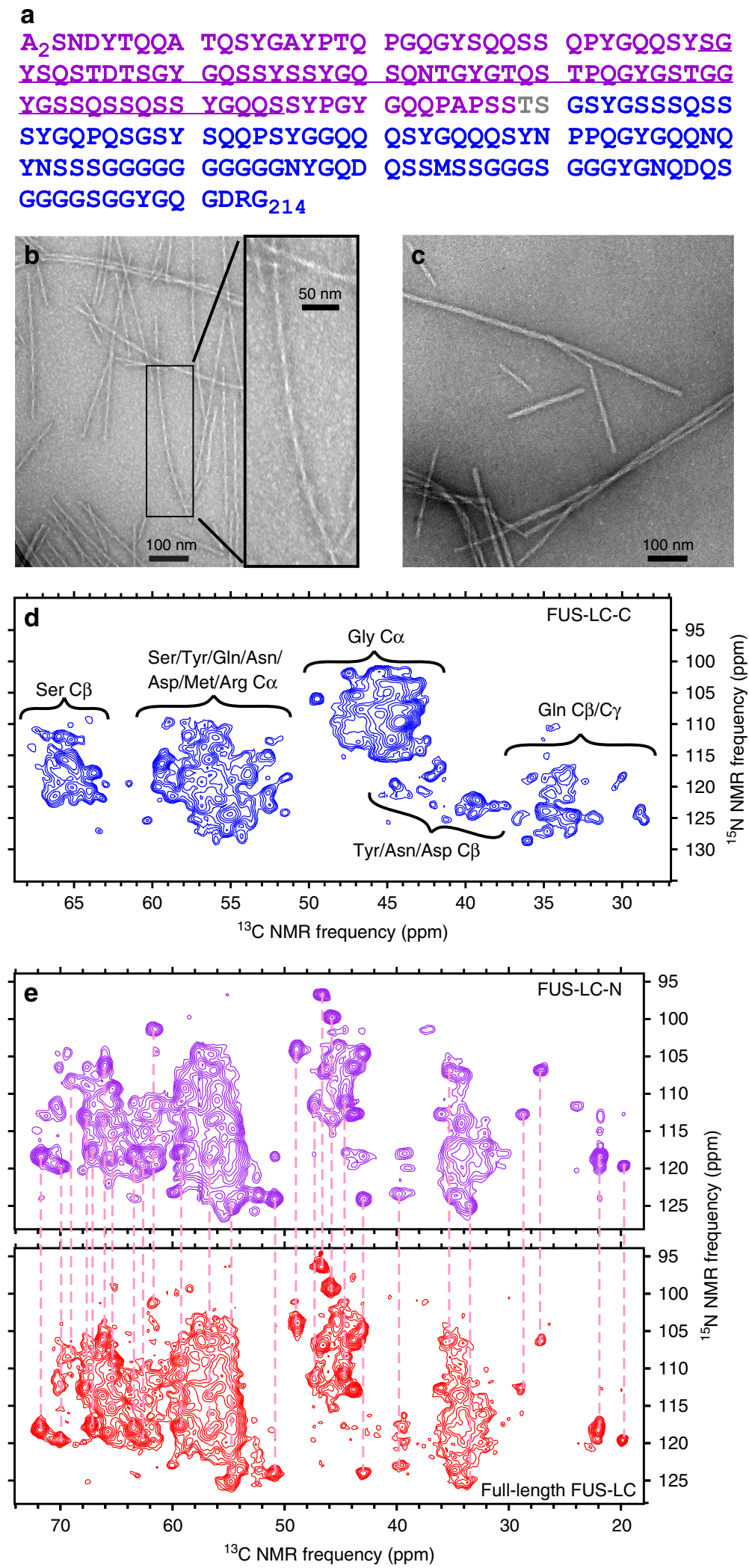

about the fibril growth direction for particles extracted from the same fibril segment ${ }^{36}$. Conditions are given in Table 1. Morphologically uniform, rapidly twisting fibrils were observed in cryo-EM images (Fig. $2 \mathrm{a}$ ). $\beta$-Strands with the expected spacing of about $4.8 \AA$ were clearly observed in $2 \mathrm{D}$ class images (Fig. $2 \mathrm{~b}$ ). Three-dimensional (3D) helical reconstruction followed by $3 \mathrm{D}$ refinement with pseudo- $2_{1}$ symmetry yielded a final density map with $2.62 \AA$ average resolution ${ }^{32}$, based on the standard Fourier shell correlation criterion (Fig. 2c and Supplementary Fig. 3). In calculations without symmetry in the helical repeat unit, the final density map was nearly the same but with $2.70 \AA$ resolution (Supplementary Fig. 3). Calculations in which $\mathrm{C}_{2}$ symmetry was 
Fig. 1 Fibril formation by full-length FUS-LC and by its $\mathbf{N}$-terminal and the C-terminal halves. a Full-length FUS-LC sequence, residues 2-214. Segments in purple and blue lettering are FUS-LC-N and FUS-LC-C, respectively. The segment that forms the structurally ordered core of full-length FUS-LC fibrils is underlined. b Negative-stain TEM image of FUS-LC-C fibrils. Inset shows the rapidly twisting morphology. The same fibril morphology was observed in 53 images. c Negative-stain TEM image of FUS-LC-N fibrils. The same fibril morphologies were observed in 20 images. d 2D NCACX ssNMR spectra of FUSLC-C fibrils, with residue-type assignments of cross-peak clusters. e 2D NCACX ssNMR spectra of uniformly ${ }^{15} \mathrm{~N}^{13} \mathrm{C}$-labeled FUS-LC-N fibrils (purple), and full-length FUS-LC fibrils (red). Vertical dashed lines, all with the same length, connect corresponding cross-peaks in the two spectra. All fibril samples were uniformly ${ }^{15} \mathrm{~N},{ }^{13} \mathrm{C}$-labeled. Contour levels in all $2 \mathrm{D}$ spectra increase by successive factors of 1.3 and were set to show approximately the same number of levels below the maximum signals in all spectra.

\begin{tabular}{|ll}
$\begin{array}{l}\text { Table } 1 \text { Cryo-EM data collection and density map } \\
\text { reconstruction parameters. }\end{array}$ \\
Data collection & \\
\hline Microscope & Titan Krios \\
Camera & Gatan K2 Summit \\
Voltage & $300 \mathrm{kV}$ \\
Magnification & $\times 130,000$ \\
Defocus range & $-1.0 \mu \mathrm{m}$ to $-2.5 \mu \mathrm{m}$ \\
Pixel size & $1.07 \AA$ \\
Exposure time (s) & $6 \mathrm{~s}$ \\
Electron dose & $47 \mathrm{e}^{-} / \AA^{2}$ \\
Reconstruction, EMDB code EMD-22169 & \\
Box size & $400 \mathrm{pixels}$ \\
Interbox distance & $35.89 \AA$ \\
Number of initial particles & 499,206 \\
Number of final particles in 3D reconstruction & 275,520 \\
Symmetry imposed & $\mathrm{Pseudo-2}$ \\
Helical rise/helical twist & $2.44 \AA / 178.94^{\circ}$ \\
Map resolution & $2.62 \AA$ \\
Map sharpening B-factor & $-83.2 \AA^{2}$ \\
\hline
\end{tabular}

imposed resulted in a density map with a similar cross-section, but with unphysical connections between neighboring repeats along the fibril growth direction.

Helical rise and twist parameters for the density map in Fig. 2c are $2.44 \AA$ and $178.94^{\circ}$, repsectively. The left-handed twist of the density map is consistent with atomic force microscope (AFM) height images (Supplementary Fig. 2d), which show left-handed asymmetry at crossovers. The $414.3 \AA$ crossover period of the density map is in good agreement with the average crossover periods of $432 \AA$ and $440 \AA$ in AFM and TEM images, respectively (Supplementary Fig. 2e).

Assuming that the repeat unit is one complete FUS-LC-C molecule (including both ordered and disordered segments), the density map implies $\mathrm{MPL}=41.1 \mathrm{kDa} / \mathrm{nm}$, in good agreement with the dark-field TEM result. Cross-sectional views of the density map show that, within each cross- $\beta$ subunit (purple and cyan in Fig. 2c), structurally ordered portions of FUS-LC-C molecules adopt U-shaped conformations, with two extended segments connected by a sharp bend or loop. Each cross- $\beta$ subunit contains two layers of in-register parallel $\beta$-sheets.

Molecular model for the FUS-LC-C fibril core. The density map from cryo-EM shows that $>50 \%$ of the residues in the structurally ordered core have bulky side chains, and that these residues occur sequentially, implying that the Ser/Gly-rich segments of FUS-LCC (residues 163-175, 182-193, and 199-207) are not in the fibril core. We therefore focused on residues 111-160 in our attempts to fit the FUS-LC-C sequence into the cryo-EM density map for the fibril core. The clearly resolved side-chain densities could only be fit with a model comprised of residues 112-150, with residues 112-127 and 132-150 forming the outer and inner extended segments, respectively (Fig. 3a). This model accounts for nearly all of the high-density regions of the map (Supplementary Fig. 4) and is consistent with dynamical information from NMR measurements described below. In contrast, models that comprised other segments of FUS-LC-C resulted in poor agreement with the density map, with multiple empty side-chain densities and side chains of the molecular models extending outside of the density (Supplementary Fig. 5).

Corrugations of backbone density align with the directions of backbone carbonyl groups in $\beta$-strand segments (Fig. $3 b$ ). If we replace the left-handed density map with its right-handed mirror image, backbone density corrugations no longer align with backbone carbonyl group directions in the molecular model that fits the mirror-image density (Supplementary Fig. 6). Thus, both molecular modeling and AFM images (Supplementary Fig. 2d) support a left-handed twist for FUS-LC-C fibrils.

The molecular model in Fig. 3a was created initially in $\operatorname{Coot}^{37}$, then refined by simulated annealing in Xplor-NIH ${ }^{38}$, using the density map as a restraint on atomic positions (probDistPot potential in Xplor-NIH). Conditions are given in Table 2. Structure bundles generated by multiple independent Xplor$\mathrm{NIH}$ calculations ${ }^{32}$ show that the molecular conformation is defined more precisely than the nominal resolution of the density map would indicate (Supplementary Fig. 7a-c). In structure bundles calculated with or without an additional statistical potential to restrain side-chain conformations (TorsionDB potential in Xplor-NIH), the root-mean-squared-displacement (RMSD) values for all backbone atoms of residues 113-149 are $0.30 \AA$ and $0.26 \AA$, respectively. RMSD values for all nonhydrogen atoms of residues $113-149$ are $0.79 \AA$ and $0.75 \AA$, respectively. The fact that many side-chain conformations are uniquely determined, so that the all-atom RMSD is substantially less than $2.63 \AA$, is attributable of the asymmetric shapes of the side-chain densities (Supplementary Fig. 7d).

The molecular model shows that residues 113-122, 135-136, and 139-149 form the $\beta$-strands of in-register parallel $\beta$-sheets. Here, $\beta$-strands are defined to be segments in which backbone amide and carbonyl groups form intermolecular hydrogen bonds along the fibril growth direction and successive amino acid side chains are on alternating faces of the $\beta$-sheets. Residues 123-134 adopt an irregular conformation that allows the protein chain to fold back on itself. Non- $\beta$-strand conformations at G137 and G138 break residues 135-149 into two discontinuous $\beta$-strands, although this segment remains fully extended.

Side chains of most Gln residues in residues 112-150 are buried within the FUS-LC-C fibril core, with the sole exception of Q124. In contrast, the majority of Ser side chains are exposed on the surface of the structurally ordered core, interacting with solvent or possibly with disordered segments in residues 151-214. Thus, burial of Gln side chains appears to be a factor that determines the core structure. The larger number of glutamines in residues 111-150 (11), compared with residues 151-214 (9), may contribute to the thermodynamic preference for residues $112-150$ as the core-forming segment of FUS-LC-C. The smaller number of glycines in residues 111-150 (7 vs. 29) is also expected to be an important factor. 

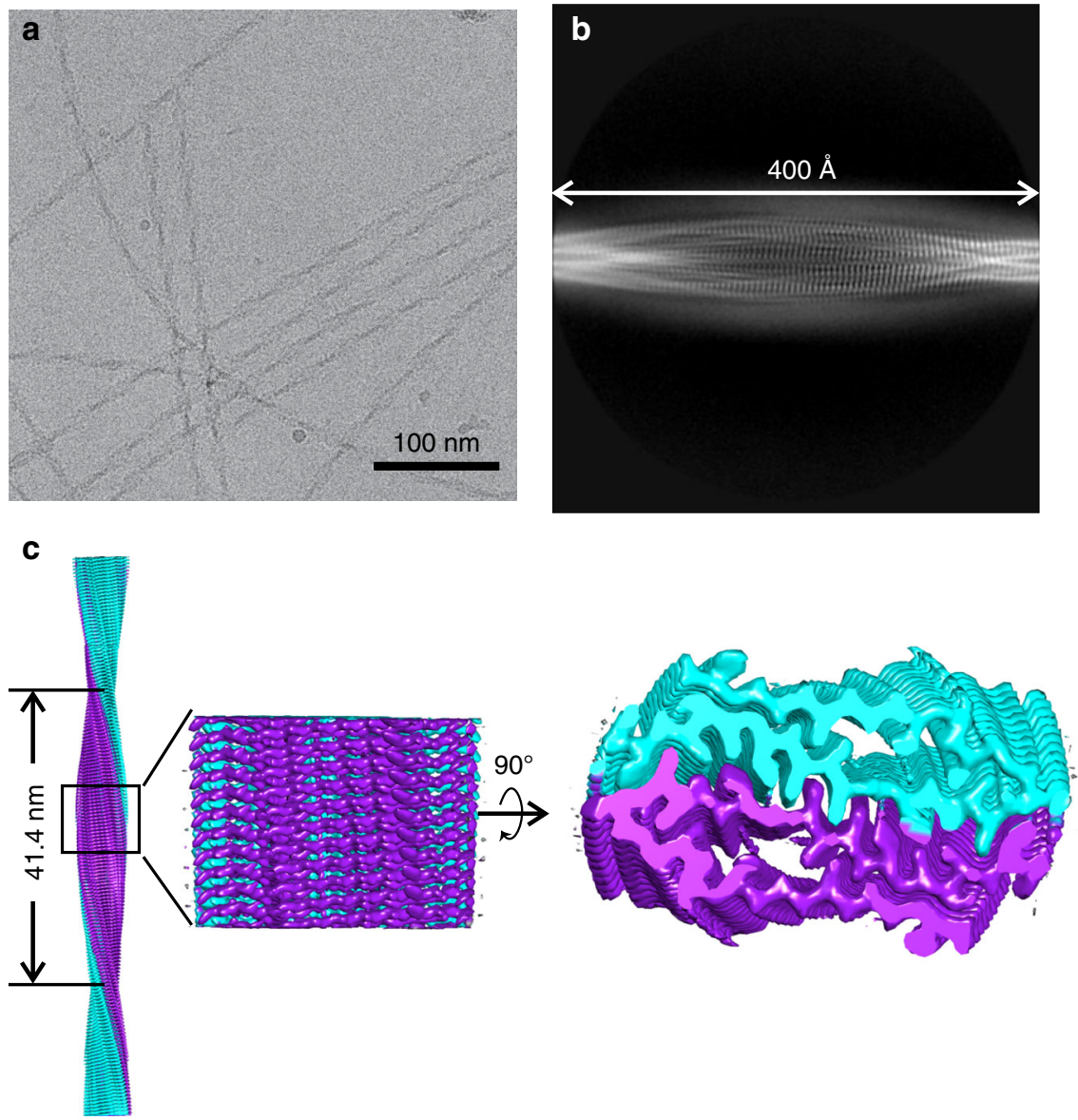

Fig. 2 Cryo-EM density map of FUS-LC-C fibrils. a Representative cryo-EM image of FUS-LC-C fibrils. $\mathbf{b}$ Example of a 2D class average image, nearly spanning one crossover period. c 3D density map reconstruction from 275,520 particles. Nominal resolution is $2.63 \AA$. The density has a left-handed twist and is reconstructed with quasi- $2_{1}$ symmetry, defined by a helical rise of $2.44 \AA$ and twist of $178.94^{\circ}$. Densities for the two cross- $\beta$ subunits are colored cyan and purple.
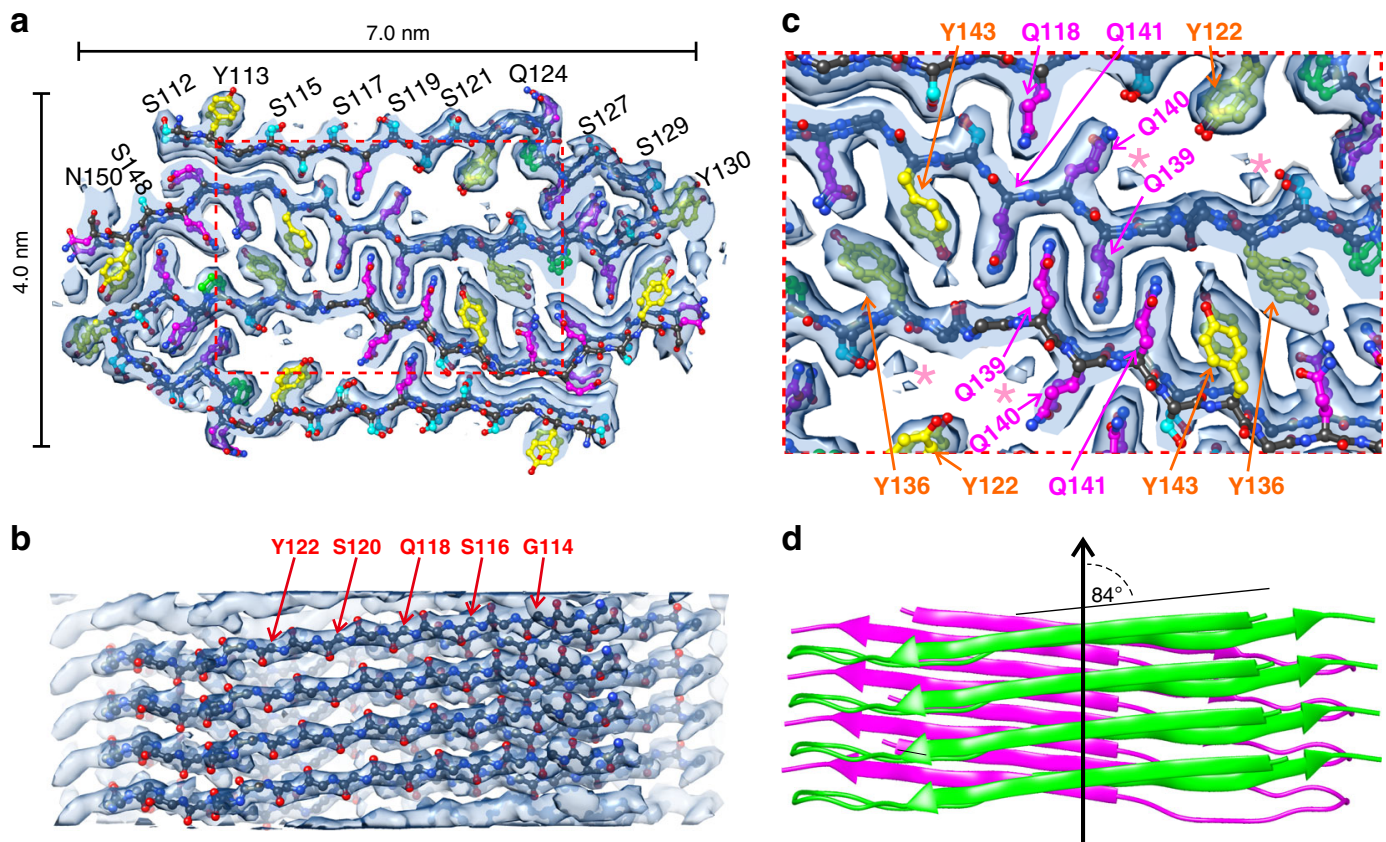

Fig. 3 Molecular structural model for the FUS-LC-C fibril core, consisting of residues 112-140. a Cross-sectional view, with side-chain carbon atoms of Ser, Tyr, Gln, and Pro residues colored cyan, yellow, magenta, and green, respectively. $\mathbf{b}$ Side view, showing that the directions of backbone carbonyl groups in the molecular model align with backbone corrugations in the density map, consistent with the left-handed twist of the density map. $\mathbf{c}$ Expanded view of the interface between cross- $\beta$ subunits. Pink asterisks indicate density attributable to ordered water molecules. $\mathbf{d}$ Cartoon representation of the molecular model, illustrating the $12^{\circ}$ angle between $\beta$-strands of the two cross- $\beta$ subunits (green and magenta) and $84^{\circ}$ angle to the fibril growth direction (black arrow). 


\begin{tabular}{|ll|}
\hline $\begin{array}{l}\text { Table } 2 \text { Xplor-NIH potentials and structure validation } \\
\text { statistics. }\end{array}$ \\
Xplor-NIH potentials \\
\hline Potential & \\
probDistPot (cryo-EM density map) & Scale factor \\
posDiffPot (2.5 $\AA$ bound, backbone atoms only) & 5.0 \\
NCS (conformational equivalence) & 10,000 \\
DistSymmpot (translational symmetry) & 100 \\
TorsionDB & 100 \\
HBDB & $0.002-2.0$ \\
RepelPot & Default \\
BOND & $0.004-4.0$ \\
ANGL & Default \\
IMPR & $0.4-1.0$ \\
Structure validation statistics, PDB code 6XFM & $0.1-1.0$ \\
MolProbity clash score & \\
Ramachandran favored & 2.0 \\
Ramachandran allowed & $93.0 \%$ \\
Ramachandran outliers & $7.0 \%$ \\
RMSD of bonds & $0.0 \%$ \\
RMSD of angles & $0.01 \AA$ \\
Number of non-hydrogen atoms & $1.50^{\circ}$ \\
Number of residues & 2360 \\
\end{tabular}

An expanded view of the central region of the fibril core (Supplementary Fig. 3) shows a classic steric zipper interdigitation of Gln side chains ${ }^{39}$, both within each cross- $\beta$ subunit (Q118 and Q140) and in the interface between subunits (Q139 and Q141). Amide groups of Gln side chains can form linear hydrogen-bonded chains, i.e., polar zippers ${ }^{40}$, which stabilize the in-register, parallel cross- $\beta$ organization (together with backbone hydrogen bonds). The interface between subunits also includes contacts between aromatic side chains of Y136 and Y143, possibly creating favorable $\pi$-stacking interactions ${ }^{41}$. Along the fibril growth direction, $\pi$-stacking interactions of Tyr side chains may also contribute to the stability of the in-register, parallel cross- $\beta$ structure ${ }^{42}$. Additional aspects of interactions that stabilize the FUS-LC-C core structure are discussed below.

The angle between $\beta$-strands and the fibril growth direction is about $84^{\circ}$ (Fig. 3d). This angle, together with the quasi-2 symmetry, creates a complex pattern of contacts between the two cross- $\beta$ subunits. For example, if the Q139 side chain of molecule $j$ in the first subunit is surrounded by Q139 and Q141 side chains of molecules $k$ and $k+1$ in the second subunit, then the Q145 side chain of molecule $j$ is near Y136 side chains of molecules $k+1$ and $k+2$, whereas the Q132 side chain of molecule $j$ is near Y149 side chains of molecules $k$ and $k-1$. Thus, each molecule in one subunit interacts with four molecules in the other subunit. The complexity of these inter-subunit interactions contributes to the stability of the FUS-LC-C core structure in the MD simulations discussed below and presumably affects the kinetics and mechanism of fibril growth.

The C-terminal segment of FUS-LC-C is dynamically disordered. To identify dynamically disordered residues, we acquired one-dimensional (1D) ${ }^{13} \mathrm{C}$ and $2 \mathrm{D}^{1} \mathrm{H}-{ }^{13} \mathrm{C}$ NMR spectra of uniformly ${ }^{15} \mathrm{~N},{ }^{13} \mathrm{C}$-labeled FUS-LC-C fibrils at $24{ }^{\circ} \mathrm{C}$ using the Insensitive Nuclei Enhanced by Polarization Transfer (INEPT) technique for scalar coupling-driven ${ }^{1} \mathrm{H}_{-}{ }^{13} \mathrm{C}$ polarization transfers $^{43}$. Signals that appear in the INEPT spectra can only arise from segments of FUS-LC-C that execute large-amplitude motions on sub-microsecond timescales ${ }^{21,44,45}$. The $1 \mathrm{D}$ and $2 \mathrm{D}$ INEPT spectra (Fig. 4a, b) show strong signals near random-coil ${ }^{1} \mathrm{H}$ and ${ }^{13} \mathrm{C}$ chemical shifts of Ser, Tyr, Gln, Asn, Asp, Met, and
Arg residues ${ }^{46}$. Signals with chemical shifts of Pro residues are weak. The presence of Arg and Asp signals, and the weakness of Pro signals indicate that the dynamically disordered segment begins after residue 150, consistent with the molecular model developed above.

For spectra in Fig. 4, the INEPT pulse sequence has the form $90_{\mathrm{X}, \mathrm{H}}-\tau_{1}-180_{\mathrm{X}, \mathrm{H}} 180_{\mathrm{X}, \mathrm{C}}-\tau_{1}-90_{\mathrm{Y}, \mathrm{H}} 90_{\mathrm{Y}, \mathrm{C}}-\tau_{2}-180_{\mathrm{X}, \mathrm{H}} 180_{\mathrm{X}, \mathrm{C}}-\tau_{2}$. The maximum peak area for the Gly $\mathrm{C}_{\alpha}$ signal spectra occurs at $\tau_{1, \max } \approx 1.4 \mathrm{~ms}$ and $\tau_{2, \max } \approx 0.7 \mathrm{~ms}$, whereas the other $\mathrm{C}_{\alpha}$ peaks have their maximum total area at $\tau_{1, \max } \approx 1.2 \mathrm{~ms}$ and $\tau_{2, \max } \approx 1.2$ ms (Fig. 4c). Assuming transverse spin relaxation times $T_{2 \mathrm{H}}$ and $T_{2 \mathrm{C}}$ for ${ }^{1} \mathrm{H}_{\alpha}$ and ${ }^{13} \mathrm{C}_{\alpha}$ nuclei, the peak areas are expected to be proportional to $\sin \left(2 \pi J \tau_{1}\right) \sin \left(2 \pi k J \tau_{2}\right) \exp \left(-\frac{2 \tau_{1}}{T_{2 \mathrm{H}}}\right) \exp \left(-\frac{2 \tau_{2}}{T_{2 \mathrm{C}}}\right)$, where $J \approx 140 \mathrm{~Hz}$ is the ${ }^{1} \mathrm{H}_{\alpha^{-}}{ }^{13} \mathrm{C}_{\alpha}$ scalar coupling constant, with $k$ being the number of ${ }^{1} \mathrm{H}_{\alpha}$ nuclei (i.e., $k=2$ for Gly $\mathrm{C}_{\alpha} ; k=1$ for other $\mathrm{C}_{\alpha}$ sites). The relaxation times are then $T_{2 \mathrm{H}}=$ $\tan \left(2 \pi J \tau_{1, \max }\right) /(\pi J)$ and $T_{2 \mathrm{C}}=\tan \left(2 \pi k J \tau_{2, \max }\right) /(k \pi J)$. From these expressions, we estimate that the average relaxation times for Gly residues are $T_{2 \mathrm{H}} \approx 6.4 \mathrm{~ms}$ and $T_{2 \mathrm{C}} \approx 3.2 \mathrm{~ms}$, and transverse spin relaxation reduces the maximum ${ }^{13} \mathrm{C}_{\alpha}$ signal of Gly residues in the 1D INEPT spectra by a factor of 0.37 relative to the ideal maximum value. For other residues, $T_{2 \mathrm{H}} \approx T_{2 \mathrm{C}} \approx 4.0 \mathrm{~ms}$, and transverse spin relaxation reduces their maximum ${ }^{13} \mathrm{C}_{\alpha}$ signals by a factor of 0.23 . Figure $4 \mathrm{c}$ shows that the ratio of maximum $1 \mathrm{D}$ INEPT signals for Gly and non-Gly residues is 0.42 . Taking into account the signal reduction factors, the ratio of dynamically disordered Gly residues in FUS-LC-C fibrils to dynamically disordered non-Gly residues is approximately $(0.23 / 0.37) \times$ $0.42=0.26$. In other words, Gly residues represent about 0.26 / $(1+0.26)=21 \%$ of the dynamically disordered residues. As Gly residues constitute $45 \%$ of residues $151-214$ (Fig. 1a), only a subset of these disordered residues execute motions with sufficiently large amplitude on sufficiently short timescales to contribute to the INEPT spectra.

Thirteen Ser residues are contained within the core-forming segment of FUS-LC-C, in rough agreement with the number of resolved or partially resolved Ser $\mathrm{C}_{\beta}$ cross-peaks in Fig. $1 \mathrm{~d}$. However, only six Gly residues are contained within the coreforming segment, whereas the $2 \mathrm{D}{ }^{15} \mathrm{~N}-{ }^{13} \mathrm{C}$ ssNMR spectrum shows signals from at least 14 inequivalent Gly residues. This observation is consistent with the conclusion from INEPT spectra that only a subset of the Gly residues are dynamically disordered.

Internal water and stabilizing interactions revealed by MD simulations. Each cross- $\beta$ subunit of the FUS-LC- $C$ fibril core contains a pore defined by side chains of Y122, S135, and Q140 and by Gly residues 137 and 138 (Fig. 3a). To investigate the properties of water within this pore, to identify other locations for internal water molecules, and to gain additional insights into interactions that stabilize the FUS-LC-C core structure, we performed all-atom MD simulations on five repeats of the $21_{1}$-symmetric structure (i.e., ten copies of FUS-LC-C residues 112-150) in explicit solvent at $303 \mathrm{~K}$. Two trajectories were analyzed from calculations with NAMD software ${ }^{47}$, one for $400 \mathrm{~ns}$ in which position constraints were applied to all $\mathrm{C}_{\alpha}$ sites, to ensure that the core structure did not disassemble during the simulation, the other for $500 \mathrm{~ns}$ without position constraints (see Supplementary Movies 1 and 2). With constraints, the backbone RMSD of the entire protein assembly was $\sim 0.8 \AA$ relative to the initial configuration throughout the $400 \mathrm{~ns}$ trajectory. Even without constraints, the core structure remained intact over the $500 \mathrm{~ns}$ trajectory, with the backbone RMSD of the six central chains remaining below $2.3 \AA$ relative to the initial configuration (Supplementary Fig. $8 \mathrm{a}$ ). We attribute the resistance of the core 

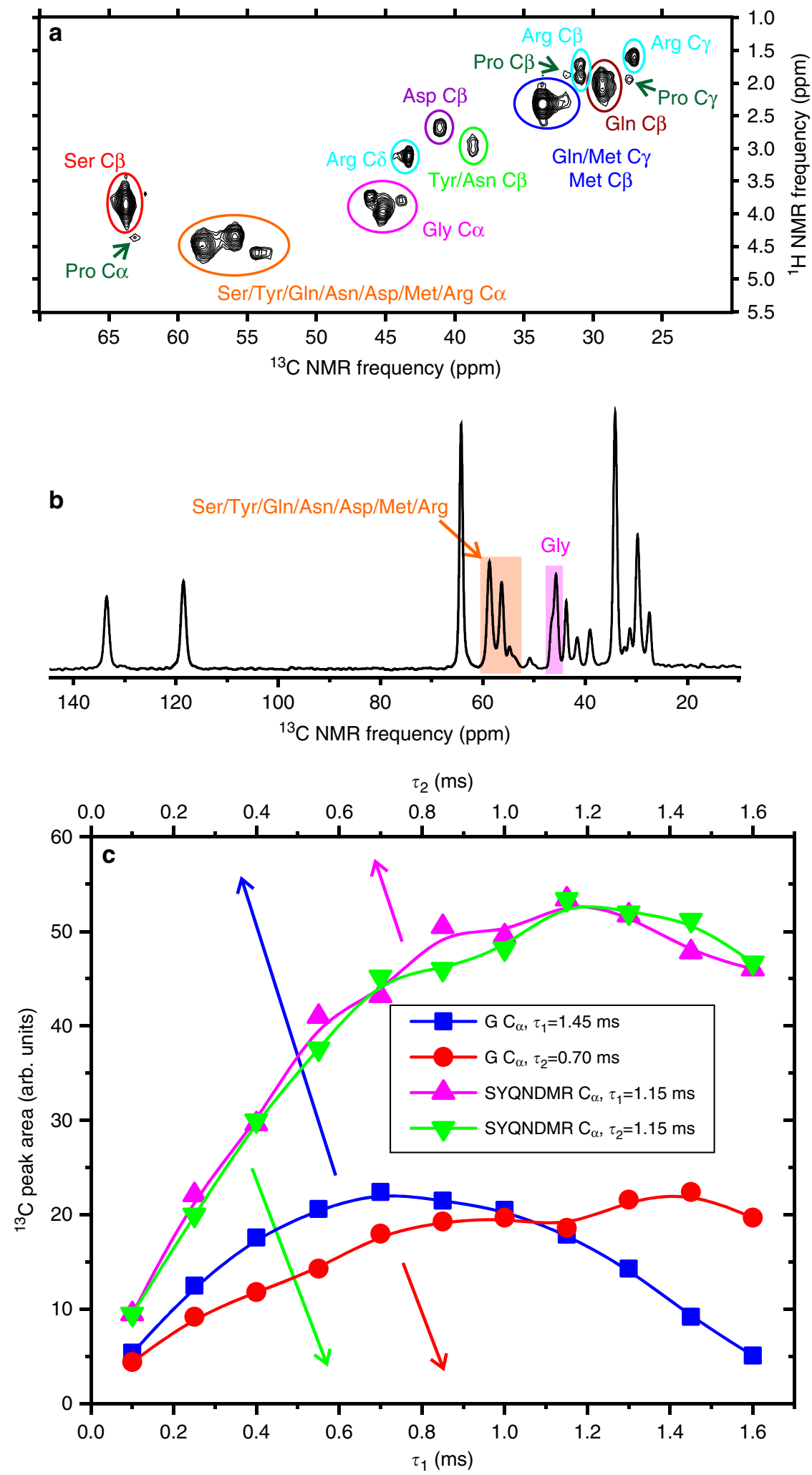

Fig. 4 Detection of dynamically disordered segments by NMR. a Aliphatic region of the $2 \mathrm{D}{ }^{1} \mathrm{H}-{ }^{13} \mathrm{C}$ INEPT spectrum of uniformly ${ }^{15} \mathrm{~N},{ }^{13} \mathrm{C}-$ labeled FUS-LC-C fibrils. Residue-type assignments of cross-peaks are based on the known random-coil ${ }^{1} \mathrm{H}$ and ${ }^{13} \mathrm{C}$ chemical shifts of each residue type. $\mathbf{b} 1 \mathrm{D}{ }^{13} \mathrm{C}$ INEPT spectrum. $C_{\alpha}$ signals of Gly residues (pink) and other residues (orange) are highlighted. $\mathbf{c}$ Dependences of $C_{\alpha}$ peak areas in $1 D$ INEPT ${ }^{13} C$ spectra on polarization transfer periods $\tau_{1}$ and $\tau_{2}$, for Gly residues (red and blue symbols, respectively) and other residues (green and pink symbols, respectively). Solid lines are guides to the eye. 


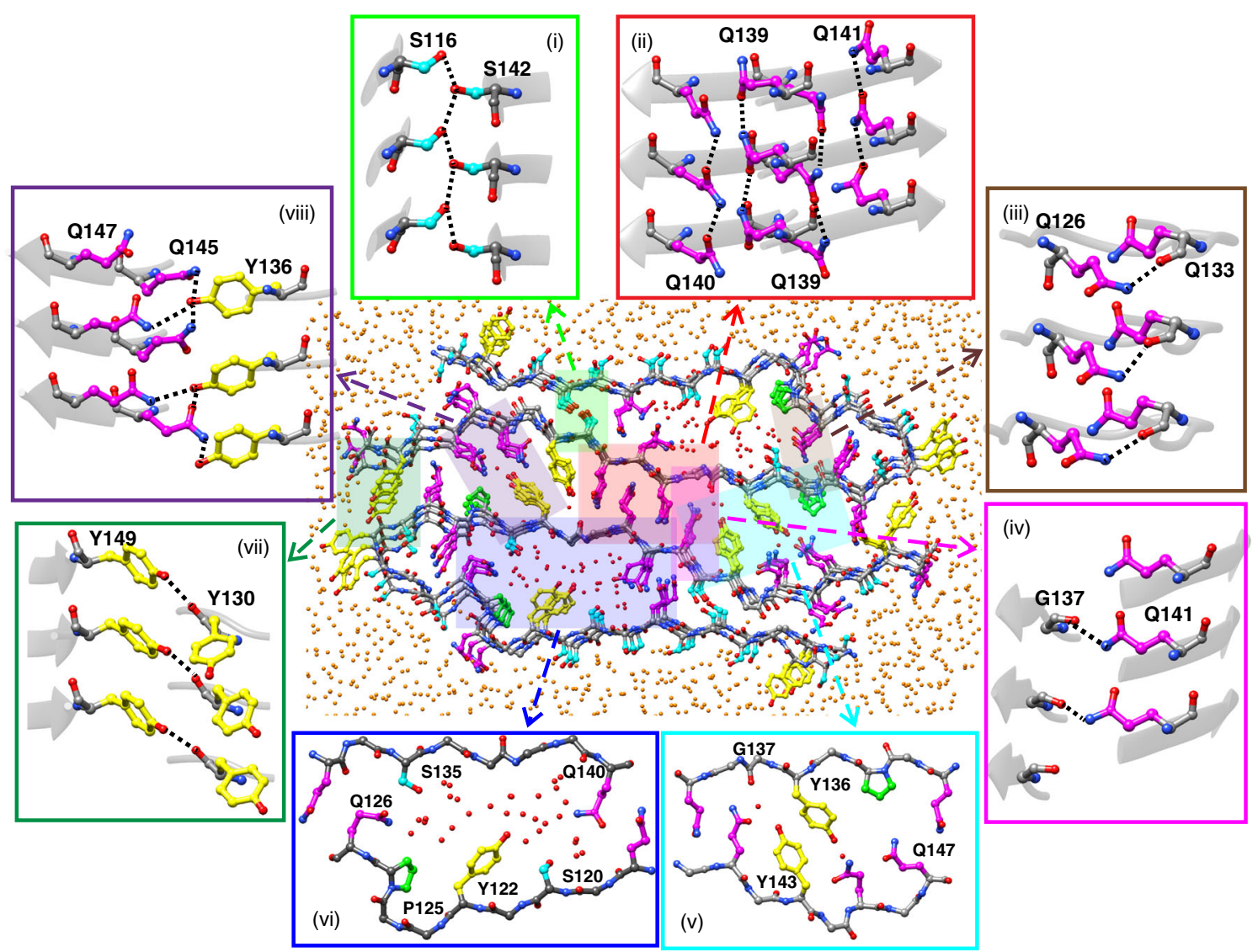

Fig. 5 Internal water and side-chain interactions in the FUS-LC-C fibril core, from all-atom molecular dynamics simulations in explicit solvent. A crosssectional slab that includes three repeats of the cryo-EM-based structure is shown after $400 \mathrm{~ns}$ of simulated dynamics, with position constraints on backbone $\mathrm{C}_{\alpha}$ atoms. Side-chain carbon atoms of Ser, Tyr, Pro, and Gln or Asn residues are shown in cyan, yellow, green, and magenta, respectively. Oxygen atoms of internal and external water molecules are shown in red and orange, respectively. Significant features include (i) S116-S142 side-chain hydrogenbonding chains along the fibril growth direction, (ii) intermolecular Gln-GIn polar zipper interactions along the growth direction, (iii) hydrogen bonds between Q126 side-chain amide and Q133 backbone carbonyl groups, (iv) hydrogen bonds between Q141 side-chain amide and G137 backbone carbonyl groups, (v) isolated internal water molecules, (vi) water within internal pores, lined with side chains of Ser, Tyr, and Gln residues, (vii) hydrogen bonds between Y149 side-chain hydroxyl and Y130 backbone carbonyl groups, and (viii) hydrogen bond networks involving side chains of Y136, Q147, and Q145.

structure to structural distortions, despite the fact that $40 \%$ of FUS-LC-C molecules in these MD simulations were located at the fibril ends, to the complex pattern of inter-subunit interactions discussed above.

In the constrained MD simulation, residues 113-122, 135-136, and 139-149 maintained their $\beta$-strand character throughout the trajectory. In the unconstrained MD simulation, fraying occurred at the $\mathrm{N}$ - and $\mathrm{C}$-terminal ends, so that the stable $\beta$-strand segments became residues 115-122, 135-136, and 139-147. Lefthanded helicity persisted throughout the unconstrained simulation, with a final value of approximately $-2.5^{\circ}$ per $4.82 \AA$ repeat.

As shown in Fig. 5, water molecules filled the main pores of the two cross- $\beta$ subunits (Fig. 5, vi). Isolated water molecules also entered the core at other locations, especially near hydroxyl and amide groups of Tyr and Gln side chains (Fig. 5, v). The density of internal water equilibrated within $0.7 \mathrm{~ns}$ in the unconstrained trajectory (Supplementary Fig. 8b). All internal water molecules were mobile on the nanosecond time scale (Supplementary Fig. 8c), but average times for internal water molecules to move $10 \AA$ were at least a factor of 10 greater than for external, bulk water (Supplementary Fig. 8d). Occupancy of hydrogen bonds to internal water was greatest for side-chain hydroxyl and amide groups around the main pores (S120, Y122, S135, and Q140), but was also relatively large for Q118, Q126, Y136, and Y143 (Supplementary Fig. 9a). Density attributable to partially ordered water molecules is observed near side chains of Q140 and S135 in the cryo-EM density map (Fig. 3c), consistent with the MD simulations.

Previous studies based on crystallography 48,49 and $s \mathrm{NMR}^{30}$ have identified side-chain hydrogen bonds as likely stabilizing interactions for cross- $\beta$ assemblies formed by LC sequences. Several distinct types of side-chain hydrogen bonds were apparent in our MD simulations. Most surprising was the observation of highly occupied sidechain-to-backbone hydrogen bonds between $\beta$-sheet layers (Fig. 5, iii, iv, and vii), including hydrogen bonds between side-chain amide groups of Q126 or Q141 and backbone carbonyl groups of Q133 or G137, respectively, and side-chain hydroxyl groups of Y149 and backbone carbonyl groups of Y130. Sidechain-to-backbone hydrogen bonds were found both within each cross- $\beta$ subunit (Supplementary Fig. 9b) and between subunits (Supplementary Fig. 9c). Sidechain-to-sidechain hydrogen bonds between $\beta$-sheet layers were also observed (Fig. 5, $\mathrm{i}$ and viii), including intrasubunit hydrogen bonds beween hydroxyl groups of S116 and S142 (Supplementary Fig. 9b) and inter-subunit hydrogen bonds between hydroxyl groups of Y136 and amide groups of Q145 and Q147 (Supplementary Fig. 9c).

Side-chain hydrogen bonds were also observed along the the fibril growth direction (Fig. 5, i and ii). In addition to the expected polar zipper interactions among Gln side chains (Supplementary 
Fig. 9d) and among N150 side chains, these included intermolecular hydrogen bonds between side-chain hydroxyl and backbone amide groups of S131, and the aforementioned S116-S142 hydrogen bonds (Supplementary Fig. 9e).

Finally, the MD simulations revealed a diverse spectrum of side-chain dynamics. All six Tyr residues were found to have strongly preferred side-chain conformations that presumably optimize $\pi$-stacking interactions along the fibril growth direction ${ }^{42}$, corresponding to side-chain torsion angles $\chi_{1} \approx-65^{\circ}$ and $\chi_{2} \approx \pm 100^{\circ}$. Tyr aromatic ring flips could then be visualized as rapid changes in the sign of $\chi_{2}$. In MD simulations with and without constraints, Y130, Y136, and Y143 did not undergo ring flips on the time scale of these simulations (Supplementary Figs. 10a and 11a, respectively). In contrast, in simulations without constraints, Y122 side chains, which project into the main water pores, did flip in the simulated trajectories, as did the solvent-exposed side chains of Y113 and Y149.

Orientations and dynamics of Gln side chains were quantified by means of a pseudo-torsion angle $\xi$, defined by the coordinates of the following four atoms: $\mathrm{N}_{\varepsilon 2}, \mathrm{O}_{\varepsilon 1}$, and $\mathrm{C}_{\alpha}$ for a Gln residue in molecule $k$ of one cross- $\beta$ subunit, and $C_{\alpha}$ for the same residue in molecule $k+1$ of the same subunit. With this definition, if the $\mathrm{O}_{\varepsilon 1}-\mathrm{N}_{\varepsilon 2}$ direction (i.e., the direction of the side-chain amide group) is parallel or antiparallel to the fibril growth direction, $\xi$ is $\sim 0^{\circ}$ or approximately $\pm 180^{\circ}$, respectively. In MD simulations without constraints (Supplementary Fig. 10b), side-chain amide groups of Q133, Q139, and Q141 maintained constant orientations throughout the $500 \mathrm{~ns}$ trajectory. These residues participated in stable polar zippers (Supplementary Fig. 9d). The Q126 side-chain orientation was also nearly constant, but with $\xi \approx 100^{\circ}$, corresponding to stable intramolecular hydrogen bonds between Q126 side-chain amide and Q133 backbone carbonyl groups. The solvent-exposed side chain of Q124 exhibited rapid, nearly random orientational fluctuations. Other Gln side chains, including Q118, Q132, Q140, Q145, Q146, and Q147, exhibited transitions among preferred orientations, including orientations that allow polar zipper interactions $\left(\xi \approx 0^{\circ}, \pm 180^{\circ}\right)$ and orientations that allow hydrogen bonds to nearby polar side chains, to internal water molecules, or to backbone carbonyl groups $(\xi \approx$ $\left.50^{\circ}, \pm 100^{\circ}\right)$. Similar behaviors were observed in MD simulations with backbone $\mathrm{C}_{\alpha}$ restraints, indicating that the dynamics and interactions of $\mathrm{Gln}$ side chains do not depend on large-amplitude backbone motions or on large deviations of the backbone conformation from the initial cryo-EM-based molecular model.

\section{Discussion}

Comparison of FUS-LC-C and FUS-LC-N fibril structures. According to ssNMR data in Fig. 1 and Supplementary Fig. 1, the core structure in FUS-LC-N fibrils is the same as in full-length FUS-LC fibrils, which was shown previously to contain an inregister, parallel cross- $\beta$ stacking of residues $39-95$ in an approximate S-shaped conformation ${ }^{21,31}$. Results described above show that the core structure in FUS-LC-C fibrils is qualitatively different, consisting of two in-register, parallel cross- $\beta$ stacks of residues $112-150$ in $U$-shaped conformations, with quasi- $2_{1}$ symmetry about the stacking direction (i.e., the fibril growth direction). Despite their structural differences, amino acid compositions of the two cores are similar (31.6\% Ser, $21.1 \%$ Gly, $17.5 \%$ Gln, $14.0 \%$ Tyr, $10.5 \%$ Thr, 5.3\% Asn/Asp/Pro in residues 39-95 of FUS-LC; 33.3\% Ser, 28\% Gln, 15.4\% Gly, 15.4\% Tyr, 7.7\% Asn/ Pro in residues $112-150$ of FUS-LC). Potentially significant differences include the higher Gln content and the lower contents of Gly and hydroxyl-bearing residues in the FUS-LC-C core.

The FUS-LC-C core includes continuous $\beta$-strand segments with 10 -residue and 11-residue lengths, with $59 \%$ of the core- forming sequence participating in $\beta$-sheets. In contrast, the two longest $\beta$-strand segments in the FUS-LC-N core are 5 and 7 residues in length, and $40 \%$ of the core-forming sequence participates in $\beta$-sheets. The term Low-complexity AromaticRich Kinked Segments (LARKS) was introduced by Eisenberg and colleagues $^{48}$ to describe cross- $\beta$ motifs in full-length FUS-LC and other LC-domain fibrils, and to distinguish these motifs from the wider or flatter $\beta$-sheets observed in other amyloid fibrils ${ }^{50-54}$. The FUS-LC-C core structure is not obviously LARKS-like. Rather, the $\beta$-sheet formed by residues $113-122$ is quite flat. The $\beta$-sheet formed by residues $139-149$ has a crease at G144, but side chains of these residues appear tightly packed and interdigitated, as in standard steric zipper structures ${ }^{39}$.

Factors that determine the structure of full-length FUS-LC fibrils. The experimental fact that full-length FUS-LC fibrils have the FUS-LC-N core rather than the FUS-LC-C core requires explanation. One possibility is that a core formed by residues 39-95 is simply more stable than a core formed by residues 112-150. Measurements of the optical absorbance of supernatants from pelleted fibril suspensions (see "Methods" in Supplementary Information), indicate equilibrium solubilities of $4.5 \pm 1.3 \mu \mathrm{M}$ for FUS-LC-C and $2.7 \pm 1.7 \mu \mathrm{M}$ for FUS-LC-N under our experimental conditions (mean $\pm \mathrm{SD}$, three independent experiments). Solubility ratios of $1.9 \pm 0.6$ imply a difference in the free energy of fibril formation $\Delta \Delta G \approx R T \ln (1.9 \pm 0.6)=0.35 \pm 0.19 \mathrm{kcal} / \mathrm{mol}$. Thus, FUS-LC-N fibrils are apparently more stable than FUS-LC-C fibrils under our experimental conditions, suggesting that the 39-95 core is indeed preferred thermodynamically. However, the difference is small. If the relative stability of the two cores were the only factor, one might expect two distinct FUS-LC fibril structures to coexist, as observed in the case of 40-residue amyloid$\beta$ (A $\beta 40)$ fibril polymorphs with similarly small differences in thermodynamic stability ${ }^{55}$.

Kinetic factors may play a role. As described above, the FUSLC-C core contains a complex pattern of contacts between the two cross- $\beta$ subunits of the quasi- $2_{1}$-symmetric structure, with each molecule in one subunit interacting with four molecules of the other subunit. Formation of this structure may then be inherently slower than formation of the FUS-LC-N core structure, which consists of a single cross- $\beta$ unit.

Another potentially important factor is the effect of fibril formation on protein segments outside the fibril core. As the FUS-LC-N and FUS-LC-C cores contain only 57 and 39 residues, respectively, more than $70 \%$ of the FUS-LC sequence remains disordered upon fibril formation. NMR spectra show that the disordered segments, or portions thereof, execute large-amplitude motions on sub-microsecond timescales, both in FUS-LC-C fibrils (Fig. 4) and in full-length FUS-LC fibrils ${ }^{21}$. However, the close proximity of the disordered segments of neighboring molecules within an in-register, parallel cross- $\beta$ structure necessarily restricts the volume available to each disordered segment, leading to a substantial decrease in configurational entropy. A 100-residue disordered polypeptide in free solution adopts conformations characterized by a radius of gyration $r_{\mathrm{g}} \approx$ $30 \AA^{56}$. If the available volume is limited to $15 r_{\mathrm{g}}{ }^{3}$ when the same polypeptide is attached to a cross- $\beta$ fibril core, the number of accessible conformations may decrease by a factor of $100^{57}$, corresponding to a free energy contribution of $R T \ln (100) \approx 2.8$ $\mathrm{kcal} / \mathrm{mol}$

The qualitative structural differences between the FUS-LC-N and FUS-LC-C cores may lead to substantial differences in the available volumes for disordered segments in the context of fulllength FUS-LC. Specifically, the U-shaped conformation of residues $112-150$ in the FUS-LC-C core would bring $\mathrm{N}$ - 
terminal and C-terminal disordered segments in close proximity, whereas the S-shaped conformation of residues 39-95 in the FUSLC-N core does not (Supplementary Fig. 12a). The greater entropy loss of disordered segments may therefore prevent formation of full-length FUS-LC fibrils with the FUS-LC-C core. In future experiments on other segments of FUS-LC, it may be possible to demonstrate the effects of disordered segments on the identities and structures of core-forming segments, using cryo-EM and ssNMR measurements. For example, in fibrils formed by residues 2- $X$ of FUS-LC, the core structure may switch from that of FUSLC-N to that of FUS-LC-C as $X$ decreases from 214 to 150.

One might also ask why full-length FUS-LC fibrils that contain both core structures do not exist. A structure containing a central 2 -symmetric FUS-LC-C core and two flanking FUS-LC-N cores may seem plausible when viewed in cross-section (Supplementary Fig. 12b). However, the FUS-LC-C core has an inherent lefthanded twist, rotating $360^{\circ}$ in $88 \pm 8 \mathrm{~nm}$. The fact that variations in the twist period are small (Supplementary Fig. 2e) implies that untwisting the FUS-LC-N core would require work that greatly exceeds thermal energy. The centers of mass of FUS-LC-N cores in a putative triple-core structure must then follow spiral paths around the fibril growth direction (Supplementary Fig. 12c). With a twist period $L \approx 88 \mathrm{~nm}$ and a radius $R \approx 5.5 \mathrm{~nm}$, the spiral path length would be $\sqrt{L^{2}+4 \pi^{2} R^{2}} \approx 95 \mathrm{~nm}$. Consequently, the average intermolecular spacing in the FUS-LC-N cross- $\beta$ structure must increase by a factor of $95 / 88=1.08$, to $5.1-5.2$ $\AA$. The energetic cost of stretching hydrogen bonds or straining molecular conformations to accommodate the larger spacing may then preclude structures such as the one depicted in Fig. 5b.

Similar principles may affect the structure of amyloid fibrils formed by other proteins, especially fibrils formed by proteins larger than $15 \mathrm{kDa}$ and proteins with uniform amino acid compositions. Entropy loss associated with segments outside the fibril core may determine the identities and conformations of core-forming segments. The diameter of the fibril core, and hence the length of the core-forming segment, may be limited by the energetics of intermolecular hydrogen bonds and molecular conformations at the perimeter of the core.

It should also be noted that FUS-LC-N contains 18 Gly and 7 Pro residues, whereas FUS-LC-C contains 36 Gly and 4 Pro residues. The larger population of Gly residues in FUS-LC-C may contribute to the identity of the core-forming segment in FUS-LC fibrils. The fact that Gly residues are concentrated after residue 165 contributes to the selection of residues 112-150 for the core of FUS-LC-C fibrils.

Interactions within the FUS-LC-C fibril core. MD simulations described above reveal several characteristics of the FUS-LC-C fibril core that are likely to be shared with other amyloid structures. In addition to water that fills the large internal pores around Y122 side chains, isolated water molecules occupy smaller cavities, especially around the side chains of Q132 and Y143. Residence times of water in large and small cavities are similar and roughly ten times longer than outside the core (Supplementary Fig. 8).

Hydrogen-bonding interactions within the core are surprisingly diverse. Polar zipper interactions within rows of Gln side chains vary in their occupancy and stability, being highly occupied and directionally stable for Q139, Q133, and Q141, but more variable for other Gln residues (Supplementary Figs. 911). Variations in dynamics and ordering of Gln side chains may explain the variability of Gln side-chain signals in ssNMR studies of amyloid fibrils, including full-length FUS-LC fibrils ${ }^{21,30}$. Similar considerations apply to Tyr side chains, which exhibit a range of ring flip rates (Supplementary Figs. 10 and 11).
In addition to polar zippers, Gln side chains can engage in hydrogen bonds to nearby backbone sites or polar side chains. For example, the Q126-Q133 interaction (Fig. 5, iii) apparently stabilizes the $\beta$-turn between these residues, together with hydrogen bonds between S131 hydroxyls and S129 carbonyls. Other highly occupied sidechain-backbone hydrogen bonds include Y149-Y130 and Q141-G137 interactions that contribute to the interface between cross- $\beta$ subunits (Supplementary Fig. 9).

Direct sidechain-sidechain hydrogen bonds between different residues are less prevalent than sidechain-backbone hydrogen bonds. The most prominent example is the S116-S142 interaction, which apparently helps stabilize the interface between $\beta$ strand segments of a single subunit. Related examples of sidechain-backbone and sidechain-sidechain hydrogen bonds occur in cross- $\beta$ crystal structures of amyloid-forming peptides $^{39,58}$, including peptides from FUS-LC ${ }^{49,59}$. Intersubunit Ser-Ser sidechain-sidechain hydrogen bonds may exist in 2 -symmetric TDP43 fibrils $^{60}$. ssNMR data indicate sidechain-backbone and sidechain-sidechain hydrogen bonds involving Ser and Thr residues in the core of full-length FUS-LC fibrils ${ }^{30}$.

Hydrophobic interactions and salt bridges can also be important stabilizing interactions for pathogenic amyloid fibrils, as indicated by structural studies of amyloid- $\beta^{50,54,61,62}$ and $\alpha$ synuclein $^{51,63}$ fibrils, as well as for functional amyloids ${ }^{64-66}$. The absence of charged amino acid side chains and the absence of purely hydrophobic residues other than Pro within the FUS-LC-C fibril core implies that hydrophobic interactions and salt bridges do not account for the formation of FUS-LC-C fibrils.

Recurring structural motifs in amyloid fibrils. U-shaped molecular conformations and two-fold symmetry about the growth direction were reported previously in early ssNMR-based structural models for synthetic $A \beta 40$ fibrils ${ }^{67}$, as well as in a recent cryo-EM structure of fibrils formed by residues 84-178 of human $\operatorname{PrP}^{68}$. Structural similarity to the FUS-LC-C fibril core reported above is especially remarkable in light of the qualitatively different amino acid compositions of A $\beta 40$ and $\operatorname{PrP}$ (both of which contain multiple Ala, Phe, Val, Leu, Ile, and Met residues in their core-forming segments). As pointed out previously ${ }^{21}$, the S-shaped molecular conformation in the FUS-LC-N core closely resembles the conformation in a-synuclein fibril cores $^{51}$. A threefold symmetric core structure has been reported for Gln-rich Orb2 fibrils ${ }^{52}$, similar to threefold-symmetric A $\beta 40$ fibrils structures from ssNMR ${ }^{61,69}$. Apparently, recurring structural motifs in amyloid cores can be stabilized either by hydrophobic interactions or by a variety of side-chain hydrogenbonding interactions, contributing to the nearly generic propensity of polypeptide chains to self-assemble into amyloid fibrils ${ }^{70}$.

\section{Methods}

Production of FUS-LC-C and FUS-LC-N. Recombinant FUS-LC-C (residues 111-214 in Fig. 1a) with an N-terminal green fluorescent protein (GFP) tag and FUS-LC-N (residues 2-108) with an N-terminal $\mathrm{His}_{6}$ tag were expressed in BL21 (DE3) Escherichia coli cells. A linker segment ending with a caspase-3 cleavage site allowed tag-free FUS-LC-C to be produced by caspase- 3 cleavage. A linker segment ending with a Tobacco Etch Virus (TEV) cleavage site allowed tag-free FUS-LC-N to be produced by TEV cleavage. Cells were grown in $1 \mathrm{~L}$ of Luria-Bertani medium (or M9 medium containing $2 \mathrm{~g} / \mathrm{L}$ of ${ }^{13} \mathrm{C}_{6}$-glucose and $1 \mathrm{~g} / \mathrm{L}$ of ${ }^{15} \mathrm{~N}$-ammonium chloride for the ssNMR samples) containing $100 \mu \mathrm{g} / \mathrm{mL}$ ampicillin at $37^{\circ} \mathrm{C}$ with shaking at 240 r.p.m. until the optical density $\left(\mathrm{OD}_{600}\right)$ reached $0.8-1.0$. Protein expression was induced by adding $0.5 \mathrm{mM}$ isopropyl $\beta$-D-1-thiogalactopyranoside and cells were further cultured at $20^{\circ} \mathrm{C}$ overnight for GFP-FUS-LC-C or at $37^{\circ} \mathrm{C}$ for $5 \mathrm{~h}$ for $\mathrm{His}_{6}$-FUS-LC-N. After protein expression, the cells were centrifuged at $4000 \times g$ for $30 \mathrm{~min}$ at $4{ }^{\circ} \mathrm{C}$. The cell pellet was resuspended in $50 \mathrm{~mL}$ of lysis buffer (20 mM Tris- $\mathrm{HCl} \mathrm{pH} 7.4,500 \mathrm{mM} \mathrm{NaCl}, 0.5 \%$ v/v Triton X-100, $20 \mathrm{mM}$ 2-mercaptoethanol, one tablet of SigmaFASTprotease inhibitor cocktail, $0.2 \mathrm{mg} / \mathrm{mL}$ 
lysozyme, and either $2 \mathrm{M}$ urea for GFP-FUS-LC-C or $2 \mathrm{M}$ guanidine-HCl for $\mathrm{His}_{6^{-}}$ FUS-LC-N) and sonicated in an ice bath for 10 min using a Branson Model 250 sonifier at 0.4 output, $30 \%$ duty cycle, with a tapered $1 / 8$ " microtip horn. The lysed cells were centrifuged at $140,000 \times g$ for $1 \mathrm{~h}$ at $4{ }^{\circ} \mathrm{C}$. The supernatant was loaded onto a gravity flow column containing $10 \mathrm{~mL}$ of nickel NTA agarose resin (Goldbio) and the column was placed in a rotator for $30 \mathrm{~min}$ at $4{ }^{\circ} \mathrm{C}$ to allow protein binding. The column was then washed with $250 \mathrm{~mL}$ of wash buffer $(20 \mathrm{mM}$ Tris-HCl pH 7.4, $200 \mathrm{mM} \mathrm{NaCl}, 20 \mathrm{mM}$ 2-mercaptoethanol, $0.1 \mathrm{mM}$ phenylmethylsulfonyl fluoride (PMSF), $25 \mathrm{mM}$ imidazole, and either $2 \mathrm{M}$ urea for GFP-FUS-LC-C or $2 \mathrm{M}$ guanidine- $\mathrm{HCl}$ for $\mathrm{His}_{6}$-FUS-LC-N) and eluted with the elution buffer (20 mM Tris- $\mathrm{HCl}$ pH 7.4, $200 \mathrm{mM} \mathrm{NaCl}, 20 \mathrm{mM}$ 2-mercaptoethanol, $0.1 \mathrm{mM}$ PMSF, $250 \mathrm{mM}$ imidazole, and either $2 \mathrm{M}$ urea for GFP-FUS-LC-C or $2 \mathrm{M}$ guanidine- $\mathrm{HCl}$ for $\mathrm{His}_{6}$-FUS-LC-N). Ethylenediaminetetraacetic acid $(0.5 \mathrm{mM})$ was added to the eluted protein solutions.

Purified proteins were concentrated using an Amicon ultracentrifuge filter with a molecular weight cutoff of $10 \mathrm{kDa}$ to $\sim 50 \mathrm{mg} / \mathrm{mL}$. GFP-FUS-LC-C was cleaved by diluting the protein to $2 \mathrm{mg} / \mathrm{mL}$ with a buffer consisting of $20 \mathrm{mM}$ Tris- $\mathrm{HCl} \mathrm{pH}$ 7.4, $200 \mathrm{mM} \mathrm{NaCl}, 20 \mathrm{mM}$ 2-mercaptoethanol, and $0.1 \mathrm{mM} \mathrm{PMSF}$, followed by addition of caspase- 3 with a 1:1500 mass ratio of caspase-3 to GFP-FUS-LC-C. Similarly, $\mathrm{His}_{6}$-FUS-LC-N was cleaved by diluting the protein to $2 \mathrm{mg} / \mathrm{mL}$ with a buffer consisting of $20 \mathrm{mM}$ Tris- $\mathrm{HCl} \mathrm{pH} \mathrm{8.0,150} \mathrm{mM} \mathrm{NaCl,} 1 \mathrm{mM}$ dithiothreitol, followed by addition of TEV protease with a 1:200 mass ratio of TEV protease to $\mathrm{His}_{6}$-FUS-LC-N. The resulting solution was rotated at room temperature overnight. Cleaved, tag-free proteins were then purified by fast protein liquid chromatography using a Superdex 200 PG column with $20 \mathrm{mM}$ Tris- $\mathrm{HCl}$ pH 7.4, $200 \mathrm{mM} \mathrm{NaCl}, 20 \mathrm{mM}$ 2-mercaptoethanol, $0.1 \mathrm{mM}$ PMSF, and $2 \mathrm{M}$ guanidine- $\mathrm{HCl}$ for $150 \mathrm{~min}$ at a $2 \mathrm{~mL} / \mathrm{min}$ flow rate. FUS-LC-C or FUS-LC-N eluted at $115-125 \mathrm{~min}$. Purified proteins were then concentrated to $\sim 50 \mathrm{mg} / \mathrm{mL}$ in $6 \mathrm{M}$ guanidine- $\mathrm{HCl}$ using an Amicon ultracentrifuge filter with a molecular weight cutoff of $3 \mathrm{kDa}$ and stored at $-80^{\circ} \mathrm{C}$.

Full-length FUS-LC, with the N-terminal His-tag MSYYHHHHHHDYDIPTTENLYFQGAMDP, was expressed in BL21(DE3)PLysS E. coli cells at $37^{\circ} \mathrm{C}$. After cell lysis by sonication in $50 \mathrm{mM}$ Tris- $\mathrm{HCl} \mathrm{pH} 7.5$ with $500 \mathrm{mM} \mathrm{NaCl}$, $1 \%$ Triton $\mathrm{X}-100,6 \mathrm{M}$ guanidine- $\mathrm{HCl}$, and protease inhibitor, lysed cells were pelleted at $223,000 \times g$ and $4^{\circ} \mathrm{C}$. FUS-LC was purified from the supernatant by binding to a nickel NTA agarose column in the presence of $8 \mathrm{M}$ urea, followed by elution with $20 \mathrm{mM}$ sodium phosphate $\mathrm{pH} 7.4,500 \mathrm{mM} \mathrm{NaCl}, 7.6 \mathrm{M}$ urea, and $200 \mathrm{mM}$ imidazole. Additional details were as previously described ${ }^{21}$.

Fibril formation. An initial batch of FUS-LC-C fibrils was prepared by dialyzing the protein solution at $2 \mathrm{mg} / \mathrm{ml}$ in $6 \mathrm{M}$ guanidine- $\mathrm{HCl}$ against $1 \mathrm{~L}$ of $20 \mathrm{mM}$ Tris$\mathrm{HCl}, 20 \mathrm{mM} 2$-mercaptoethanol, $0.1 \mathrm{mM}$ PMSF at $\mathrm{pH} 7.4$ overnight. The sample was then incubated at room temperature for 5-7 days. Fibril formation was verified by negative-stain TEM. Negative-stain TEM images showed that many of the FUSLC-C fibrils occurred in multi-stranded bundles. To prepare single-stranded fibril seeds for a subsequent batch of fibrils, fibrils from the initial batch were pelleted at $280,000 \times g$ for $1 \mathrm{~h}$ at $4^{\circ} \mathrm{C}$ in a Beckman Optima ultracentrifuge. After this ultracentrifugation step, single-strand fibrils were observed in the supernatant. These single-stranded fibrils were then sonicated with a Branson Model 250 sonifier and tapered $1 / 8$ " microtip horn at 0.1 output and $10 \%$ duty cycle for $10 \mathrm{~min}$, yielding short fibril fragments (i.e., seeds). Fibril growth in the subsequent batch was then initiated by adding $5 \%$ seeds to $0.4 \mathrm{mg} / \mathrm{mL}$ of monomeric FUS-LC-C, which had been dialyzed against $1 \mathrm{~L}$ of $20 \mathrm{mM}$ Tris- $\mathrm{HCl}, 20 \mathrm{mM}$ 2-mercaptoethanol, $0.1 \mathrm{mM}$ $\mathrm{PMSF}$ at $\mathrm{pH} 7.4$ overnight. This solution was then incubated at room temperature for 4 days.

Approximately 20\% of our cryo-EM images of FUS-LC-C fibrils contain minor fractions of non-fibrillar aggregates. As non-fibrillar aggregates are readily distinguished from fibrils in the manual particle picking process, they do not contribute to the density map or structural model for FUS-LC-C fibrils.

For NMR measurements, seeded fibrils, prepared as described above but using uniformly ${ }^{15} \mathrm{~N},{ }^{13} \mathrm{C}$-labeled FUS-LC-C, were pelleted at $280,000 \times g$ and $4{ }^{\circ} \mathrm{C}$ for $1 \mathrm{~h}$ in a Beckman Optima ultracentrifuge. Fibril pellets were then packed into $3.2 \mathrm{~mm}$ magic-angle-spinning (MAS) rotors by centrifugation with a swinging-bucket rotor at $70,000 \times g$ for $1 \mathrm{~h}$ at $4{ }^{\circ} \mathrm{C}$, using a home-made funnel device to hold the MAS rotor and the centrifuge tube that contained the fibril pellet.

Uniformly ${ }^{15} \mathrm{~N},{ }^{13} \mathrm{C}$-labeled FUS-LC-N fibrils for ssNMR were prepared and packed with the same procedures.

Measurements of FUS-LC-C and FUS-LC-N solubility. FUS-LC-C and FUS-LCN proteins were separately diluted to $3 \mathrm{mg} / \mathrm{mL}$ in $20 \mathrm{mM}$ Tris- $\mathrm{HCl} \mathrm{pH} \mathrm{7.5,} 200 \mathrm{mM}$ $\mathrm{NaCl}, 20 \mathrm{mM}$ 2-mercaptoethanol, $0.5 \mathrm{mM}$ ETDA, $0.1 \mathrm{mM}$ PMSF, and $6 \mathrm{M}$ guanidine- $\mathrm{HCl}$, and dialyzed overnight against $1 \mathrm{~L}$ of $20 \mathrm{mM}$ Tris- $\mathrm{HCl} \mathrm{pH} \mathrm{7.5,200}$ $\mathrm{mM} \mathrm{NaCl}, 20 \mathrm{mM}$ 2-mercaptoethanol, 0.5 mM ETDA, 0.1 mM PMSF, and 0.02\% sodium azide. The protein solutions were transferred to tubes, the protein concentrations were adjusted to $2 \mathrm{mg} / \mathrm{ml}$ with dialysis buffer, and the solutions were incubated at $24^{\circ} \mathrm{C}$ for 1 week for full fibril formation. The resulting FUS-LC-C and FUS-LC-N fibrils were pelleted at $280,000 \times g$ and $4{ }^{\circ} \mathrm{C}$ for $1 \mathrm{~h}$. After the supernatants were removed, the fibril pellets were washed with dialysis buffer, then resuspended in fresh buffer by sonication. After incubation for three days to allow equilibration of fibrillar FUS-LC-C or FUS-LC-N with monomers in solution, the fibrils were again pelleted at $280,000 \times g$ and $4{ }^{\circ} \mathrm{C}$ for $1 \mathrm{~h}$. Protein concentrations in the supernatants, representing equilibrium solubilities of FUS-LC-C or FUS-LC-N, were then determined from optical absorbance at $280 \mathrm{~nm}$. The experiments were repeated three times.

Transmission electron microscopy. For negative-stain TEM, samples were prepared on grids consisting of a $3 \mathrm{~nm}$ carbon film supported by lacey carbon on $300-$ mesh copper (Electron Microscopy Sciences LC325-Cu-CC). For dark-field TEM, samples were prepared on grids consisting of $2 \mathrm{~nm}$ carbon films on 300-mesh copper (Quantifoil S 7/2). Grids were glow-discharged immediately before use. For negative-stain images, a $10 \mu \mathrm{L}$ aliquot of fibril solution was applied to the grid. After $2 \mathrm{~min}$ adsorption to the carbon film, the grid was blotted and washed with $10 \mu \mathrm{L}$ of water for $10 \mathrm{~s}$, blotted, stained with $10 \mu \mathrm{L}$ of $2 \%$ w/v uranyl acetate for $1 \mathrm{~min}$, blotted, and dried in air. Negative-stain TEM images were obtained with a FEI Morgagni microscope, operating at $80 \mathrm{kV}$, using a side-mounted Advantage HR camera (Advanced Microscopy Techniques).

For dark-field images used in MPL measurements, $5 \mu \mathrm{L}$ of fibril solution $(0.2 \mathrm{mg} / \mathrm{mL})$ and a $5 \mu \mathrm{L}$ of tobacco mosaic virus (TMV) solution $(0.46 \mathrm{mg} / \mathrm{mL})$ were applied to a freshly glow-discharged grid. After $5 \mathrm{~min}$ of incubation, the grid was blotted, washed three times with $10 \mu \mathrm{L}$ of water for $10 \mathrm{~s}$ each, blotted, and dried in air. Dark-field images were acquired with the same FEI Morgagni microscope, operating at $80 \mathrm{kV}$, using a bottom-mounted XR-550B camera (Advanced Microscopy Techniques), with an electron beam tilt angle of $1.2^{\circ}$ and $\times 5600$ magnification. Care was taken to ensure uniform illumination of the field-of-view by the electron beam, full blockage of the direct beam by the objective aperture of the microscope, and other conditions described previously ${ }^{33}$

Dark-field images were analyzed with ImageJ software (available at http:// imagej.nih.gov/ij). Image intensities were integrated within $100 \mathrm{~nm} \times 60 \mathrm{~nm}$ rectangular areas centered on TMV rods or FUS-LC-C fibrils $\left(I_{\text {center }}\right)$, or in background regions on both sides of the TMV rods or FUS-LC-C fibrils ( $I_{\mathrm{B} 1}$ and $I_{\mathrm{B} 2}$, see Supplementary Fig. 2a). Each MPL count was calculated according to the equation MPL $=\left(I_{\mathrm{F}} / I_{\mathrm{TMV}}\right) \times 131 \mathrm{kDa} / \mathrm{nm}$, where $I_{\mathrm{F}}=I_{\text {center }}-\left(I_{\mathrm{B} 1}+I_{\mathrm{B} 2}\right) / 2$ for one fibril segment and $I_{\mathrm{TMV}}$ is the average value of $I_{\text {center }}-\left(I_{\mathrm{B} 1}+I_{\mathrm{B} 2}\right) / 2$ for many TMV rods. A total of 96 counts was used for the histogram in Supplementary Fig. 2b, with $I_{\mathrm{TMV}}$ being the average of 52 measurements. For the MPL error distribution in Supplementary Fig. 2c, error values $E$ were determined from integrated background intensities $I_{\mathrm{B}}$ according to the equation $E=\sqrt{\frac{3}{2}}\left[\left(I_{\mathrm{B}}-I_{\mathrm{B}, \text { ave }}\right) / I_{\mathrm{TMV}}\right] \times 131 \mathrm{kDa} / \mathrm{nm}$ where $I_{\mathrm{B}, \text { ave }}$ is the average of the background intensities. Gaussian fitting of MPL and error histograms was performed with Igor Pro 8.03 (WaveMetrics).

Atomic force microscopy. Tapping mode AFM was performed on a Veeco Multimode AFM instrument with Nanoscope IV controller, using a PPP NCHAuD-10 probe (Nanosensors). For AFM measurements, a $2 \mu \mathrm{L}$ aliquot of FUS-LC-C fibril solution $(0.3 \mathrm{mg} / \mathrm{mL})$ was combined with $18 \mu \mathrm{L}$ of deionized water. The diluted fibril solution was applied to a freshly cleaved mica surface, which was then blotted, washed with $10 \mu \mathrm{L}$ of water, blotted, and dried in air. AFM images were collected with a $3.12 \mathrm{~Hz}$ scan rate, 512 points/line, and 512 lines over a $1.0 \mu \mathrm{m} \times 1.0 \mu \mathrm{m}$ area.

Cryo-EM image acquisition. Quantifoil R2/2 grids (SPI Supplies) with 300 mesh were glow-discharged immediately before use. A $2.5 \mu \mathrm{L}$ aliquot of fibril solution $(0.4 \mathrm{mg} / \mathrm{mL})$ was applied to the grid with $10 \mathrm{~s}$ preblotting and $5 \mathrm{~s}$ blotting, then plunge-frozen into liquid ethane using a Leica EM GP2 plunger. Data were collected on a Titan Krios microscope, operating at $300 \mathrm{kV}$ and using $\times 130,000$ magnification. A total of 4425 micrographs was recorded with $1.07 \AA$ pixel size, 47 electrons $/ \AA^{2}$ dose, $1.0-2.5 \mu \mathrm{m}$ defocus range, and $6 \mathrm{~s}$ exposure time, using SerialEM 3.7 and AMT Image Capture Engine 6.02 software.

Cryo-EM image processing. From the 4425 micrographs, micrographs without fibrils and micrographs that contained defects or crystalline ice were manually deleted, leaving 2411 micrographs for image processing. All image processing steps were performed using RELION $3.0^{34,35}$ and a modified version of RELION 3.0, with modifications to include correlations of orientations about the fibril growth direction (i.e., $\rho$ angles) for particles that came from the same fibril segment ${ }^{36}$. Single-stranded fibrils were manually selected and a total of 499,206 particles were extracted, using 400 pixel ( $428 \AA$ ) box sizes and an interbox distance of $35.89 \AA$ (91.6\% overlap). After 14 rounds of $2 \mathrm{D}$ classification, $1132 \mathrm{D}$ classes with 406,815 particles were selected for 3D classification. Initial 3D classification was performed using a featureless cylinder as an initial model. We performed multiple rounds of $3 \mathrm{D}$ classification with the helical parameters estimated initially from the negativestain TEM images of fibrils (i.e., $4.8 \AA$ helical rise and $-2.0^{\circ}$ helical twist), then refined with the local helical symmetry search. After the initial round of 3D classification, we used in-house Matlab scripts to examine the consistency of angular alignments of particles and to fix in-plane rotation angles (i.e., $\psi$ angles) to make all particles from the same fibril segment have consistent rotational angles relative to the fibril growth direction. After three rounds of 3D classification with one class, a fourth round of $3 \mathrm{D}$ classification was performed with three classes, to include the possibility of polymorphism in the FUS-LC-C fibrils. The major class, 
containing $69 \%$ of the particles (275,520 particles), yielded the best helical density map, with 3.79 丹 resolution. The other two classes yielded lower-resolution density maps (6-7 ̊̊ resolution), which were not qualitatively different from the major class. Particles from the major class were used for further processing.

Three-dimensional auto-refinement and post-processing were then performed, improving the resolution to $3.11 \AA$. After contrast transfer function refinement and motion correction adjustments, $3 \mathrm{D}$ auto-refinement and post-processing were repeated. The resulting density map (Supplementary Fig. 3a) had 2.70 A resolution, with helical rise and twist of $4.89 \AA$ and $-2.11^{\circ}$. Examination of this density map revealed nearly perfect $2_{1}$ symmetry. Therefore, $3 \mathrm{D}$ auto-refinement and postprocessing were performed again, this time with quasi- $2_{1}$ symmetry, corresponding to initial values for helical rise and twist of $\sim 0.5 \times 4.89 \AA$ and $180^{\circ}-0.5 \times 2.11^{\circ}$. The resolution of the final density map with quasi- $2_{1}$ symmetry (Figs. 2 and 3 ) was 2.62 $\AA$, and helical rise and twist of $2.44 \AA$ and $178.94^{\circ}$, respectively (Electron Microscopy Data Bank code EMD-22169).

NMR measurements. ssNMR measurements on FUS-LC-C and FUS-LC-N fibrils were performed at $17.5 \mathrm{~T}\left(745.6 \mathrm{MHz}{ }^{1} \mathrm{H}\right.$ NMR frequency), using a Varian InfinityPlus spectrometer console and a $3.2 \mathrm{~mm}$ MAS NMR probe from Black Fox, Inc. (Tallahassee, Florida), with MAS frequencies of $12.00 \mathrm{kHz}$ (FUS-LC-C) or 16.50 $\mathrm{kHz}$ (FUS-LC-N). The spectrometer was controlled by Spinsight 4.3.2 software. 2D ${ }^{13} \mathrm{C}-{ }^{13} \mathrm{C}$ spectra were acquired with $25 \mathrm{~ms}{ }^{13} \mathrm{C}-{ }^{13} \mathrm{C}$ dipolar-assisted rotational resonance (DARR) mixing periods ${ }^{71}$ and $85 \mathrm{kHz}$ two-pulse phase-modulated (TPPM) ${ }^{1} \mathrm{H}$ decoupling ${ }^{72}$. Maximum $t_{1}$ periods were $6.40 \mathrm{~ms}$ or $3.20 \mathrm{~ms}$, with $1.5 \mathrm{~s}$ recycle delays and total data acquisition times of 10 or $18 \mathrm{~h}$, for FUS-LC-N or FUSLC-C fibrils, respectively. 2D NCACX spectra were acquired with $4.0 \mathrm{~ms}{ }^{15} \mathrm{~N}-{ }^{13} \mathrm{C}_{\alpha}$ cross-polarization periods, $25 \mathrm{~ms}{ }^{13} \mathrm{C}^{13} \mathrm{C}$ DARR mixing periods, $85 \mathrm{kHz}$ TPPM decoupling, maximum $t_{1}$ periods of $7.5 \mathrm{~ms}$ or $7.9 \mathrm{~ms}$, and total data acquisition times of 23 or $60 \mathrm{~h}$.

The ${ }^{13} \mathrm{C}$-detected $2 \mathrm{D}{ }^{1} \mathrm{H}-{ }^{13} \mathrm{C}$ INEPT spectrum of FUS-LC-C fibrils was acquired at $17.5 \mathrm{~T}$ with $12.00 \mathrm{kHz}$ MAS, using INEPT transfer periods $\tau_{1}=1.25 \mathrm{~ms}$ and $\tau_{2}=0.7 \mathrm{~ms}$, a maximum $t_{1}$ period of $10.0 \mathrm{~ms}$, a recycle delay of $2.0 \mathrm{~s}$, and a total data acquisition time of $4.2 \mathrm{~h} .{ }^{1} \mathrm{H}$ decoupling used a train of $180^{\circ}$ pulses with a 25 -step phase pattern ${ }^{73}$, with a $25 \mu \mathrm{s} 180^{\circ}$ pulse length. 1D INEPT spectra with various combinations of $\tau_{1}$ and $\tau_{2}$ were acquired in $0.15 \mathrm{~h}$ each.

ssNMR measurements on full-length FUS-LC fibrils were performed at $14.1 \mathrm{~T}$ (599.1 MHz ${ }^{1} \mathrm{H}$ NMR frequency), using a Varian InfinityPlus spectrometer console and a $3.2 \mathrm{~mm}$ MAS NMR from Varian. The MAS frequency was $12.00 \mathrm{kHz}$, TPPM decoupling fields were $85 \mathrm{kHz},{ }^{13} \mathrm{C}-{ }^{13} \mathrm{C}$ spin diffusion mixing periods were $50 \mathrm{~ms}$, and recycle delays were $1.5 \mathrm{~s}$. The $2 \mathrm{D}^{13} \mathrm{C}-{ }^{-13} \mathrm{C}$ spectrum was acquired in $22 \mathrm{~h}$ with a maximum $t_{1}$ period of $6.0 \mathrm{~ms}$. The $2 \mathrm{D}$ NCACX spectrum was acquired in $36 \mathrm{~h}$ with a maximum $t_{1}$ period of $14.9 \mathrm{~ms}$, with a $4.0 \mathrm{~ms}^{15} \mathrm{~N}-{ }^{13} \mathrm{C}_{\alpha}$ cross-polarization period.

Sample temperatures were $24 \pm 4^{\circ} \mathrm{C}$ in all NMR measurements. 2D spectra were processed with nmrPipe software ${ }^{74}$, using $0.4-0.5$ p.p.m. Gaussian apodization in both dimensions of ${ }^{13} \mathrm{C}-{ }^{13} \mathrm{C}$ and NCACX spectra of FUS-LC-N and full-length FUS-LC fibrils, 0.2 p.p.m. in the ${ }^{13} \mathrm{C}$ dimensions, 0.5 p.p.m. in the ${ }^{15} \mathrm{~N}$ dimension of ${ }^{13} \mathrm{C}^{-13} \mathrm{C}$ and NCACX spectra of FUS-LC-C fibrils, and 0.01 p.p.m. in the ${ }^{1} \mathrm{H}$ dimension and 0.2 p.p.m. in the ${ }^{13} \mathrm{C}$ dimension of ${ }^{1} \mathrm{H}-{ }^{13} \mathrm{C}$ INEPT spectra. $2 \mathrm{D}$ spectra were plotted with Sparky software (https://www.cgl.ucsf.edu/home/sparky).

Development of molecular model. As described in the main text, the bulky side chains in the density map (Fig. 3) indicated that the Gly-rich C-terminal segment of FUS-LC-C is not included in the fibril core. After examining various possibilities, we found that residues 112-150 fit well into the density map, whereas attempts to fit other segments into the density map resulted in empty side-chain densities or side chains of the model that projected outside the density (Supplementary Fig. 5). A single chain consisting of residues 112-150 was manually fit into the density for one molecule using Coot software ${ }^{37}$. Eight copies of this chain were then fit into the density map for four repeats of the $2_{1}$-symmetric dimer, using the Fit in Map function of UCSF Chimera ${ }^{75}$.

Further refinement of the molecular model was then performed with Xplor-NIH software ${ }^{38}$, using the probDistPot potential energy function to restrain all nonhydrogen atoms of the eight chains within the cryo-EM density map. Noncrystallographic symmetry (NCS potential of Xplor-NIH) was included to make the conformations of all chains nearly identical. Translational symmetry (DistSymmPot) restraints were applied to enforce translational symmetry within each of the two cross- $\beta$ subunits. A PosDiffPot potential was used to restrain the backbone atoms of each chain to within $2.5 \AA$ of their initial positions, in order to prevent each chain from hopping to the density of a neighboring chain during simulated annealing from high temperatures. Torsion angle (TorsionDB), hydrogen bond (HBDB), and standard potentials to define bond lengths and angles, improper dihedral angles, and atomic radii were used (BOND, ANGL, IMPR, and RepelPot potentials). Scale factors for all potential energy terms are summarized in Table 2 .

In the first set of Xplor-NIH calculations, ten independent structures were calculated with a probDistPot scaling factor of 10.0 , using annealing from $3000 \mathrm{~K}$ to $25 \mathrm{~K}$ in $12.5 \mathrm{~K}$ temperature decrements, with 1500 steps of torsion angle dynamics at each temperature. Annealing was followed by energy minimization in torsion angle and Cartesian space. The two lowest energy structures without violations were used as starting structures for the second set of calculations, which used the same conditions, but with a probDistPot scaling factor of 5.0. Fourteen structures without violations (except insignificant violations of the NCS potential) were deposited in the Protein Data Bank (PDB code 6XFM) and are shown in

Supplementary Fig. 7 a.

Another set of calculations (with the same two starting structures) was performed without the TorsionDB potential, to examine how the cryo-EM density map alone determines side-chain conformations. These calculations produced eight structures without violations, shown in Supplementary Fig. 7b.

MD simulations. MD simulations were performed with NAMD software ${ }^{47}$ and CHARMM22 force fields ${ }^{76}$. Five repeats of a $2_{1}$-symmetric FUS-LC-C dimer were generated from the lowest energy structure from the first set of Xplor-NIH calculations. The resulting 10 -mer was then solvated and neutralized in $100 \mathrm{mM} \mathrm{NaCl}$ in a $84.3 \AA \times 65.0 \AA \times 46.3 \AA$ box in $\mathrm{VMD}^{77}$. Constant-pressure simulations with Langevin dynamics were run at $303 \mathrm{~K}$ with periodic boundary conditions. One MD trajectory was calculated for $400 \mathrm{~ns}$, with $\mathrm{C}_{\alpha}$ atoms being constrained to their initial positions by harmonic potentials. A constraint scaling factor of 0.3 was chosen to allow moderate fluctuations of backbone coordinates in this trajectory, corresponding to a backbone RMSD of about $0.8 \AA$. Another MD trajectory was calculated for $500 \mathrm{~ns}$, without constraints of any type.

Analyses of the MD trajectories were performed with VMD software ${ }^{77}$. Using the labels $A_{1}-A_{5}$ for molecules in one cross $-\beta$ subunit and $B_{1}-B_{5}$ for molecules in the other subunit, data in Supplementary Fig. $8 \mathrm{~b}$ were obtained by counting the number of water molecules within $7.0 \AA$ of side-chain OH of Y122 or Y143 or within $7.0 \AA$ of side-chain $\mathrm{C}_{\delta}$ of Q132 or Q145 and averaging the resulting numbers for $\mathrm{A}_{3}$ and $\mathrm{B}_{3}$ (in the unconstrained trajectory). For water near the Q133, we counted the number of water molecules within $5.0 \AA$ of side-chain $\mathrm{C}_{\delta}$ of Q133 to exclude water molecules outside the fibril core. For Supplementary Fig. 8c, d, water molecules within $7.0 \AA$ of side-chain $\mathrm{OH}$ or $\mathrm{C}_{\delta}$ of $\mathrm{Y} 122, \mathrm{Y} 143, \mathrm{Q} 132$, and Q145 were selected at arbitrary time points $\mathrm{t}$ with the first $10 \mathrm{~ns}$ of the unconstrained trajectory.

For Supplementary Fig. 9, hydrogen bonds were defined by donor-acceptor distances $<3.0 \AA$ and deviations from linearity less than $20^{\circ}$. Occupancies of hydrogen bonds were defined as the fraction of frames where the hydrogen bonds were found. Data in Supplementary Fig. 9a were averaged over results for $\mathrm{A}_{3}$ and $B_{3}$. For Supplementary Fig. 9b, occupancies of hydrogen bonds between residues 112-129 and residues 131-150 were also averaged over values for $A_{3}$ and $B_{3}$. For Supplementary Fig. $9 c$, hydrogen bonds between $A_{3}$ and $B_{2}, B_{3}$, or $B_{4}$ and between $B_{3}$ and $A_{2}, A_{3}$, or $A_{4}$ were examined, and occupancies for the two cases were averaged. For Supplementary Fig. 9d, e, hydrogen bonds between $A_{3}$ and $A_{2}$ or $A_{4}$ and between $\mathrm{B}_{3}$ and $\mathrm{B}_{2}$ or $\mathrm{B}_{4}$ were examined, and occupancies for the two cases were averaged.

For Supplementary Figs. 10 and 11, time-dependent torsion angles $\chi_{2}$ of Tyr residues or pseudo-torsion angles $\xi$ of Gln residues in $\mathrm{A}_{3}$ and $\mathrm{B}_{3}$ were obtained from the unconstrained and constrained trajectories, respectively. The definition of $\xi$ is given in the caption to Supplementary Fig. 10.

Reporting summary. Further information on experimental design is available in the Nature Research Reporting Summary linked to this paper.

\section{Data availability}

The cryo-EM density map for FUS-LC-C fibrils is available from the Electron Microscopy Data Bank, code EMD-22169. Atomic coordinates for a bundle of 14 molecular structures that fit the density map are available from the Protein Data Bank, code 6XFM. The density map and coordinates are available at https://doi.org/10.2210/ pdb6XFM/pdb. The 2D ssNMR spectra are available at https://doi.org/10.17632/ ts9p355m3d.1. All other data are available from the authors upon request.

\section{Code availability}

Modified RELION 3.0 software and associated scripts for cryo-EM image processing are available from the authors upon request.

Received: 19 June 2020; Accepted: 14 October 2020; Published online: 12 November 2020

\section{References}

1. Banani, S. F., Lee, H. O., Hyman, A. A. \& Rosen, M. K. Biomolecular condensates: organizers of cellular biochemistry. Nat. Rev. Mol. Cell Biol. 18, 285-298 (2017).

2. Martin, E. W. \& Mittag, T. Relationship of sequence and phase separation in protein low complexity regions. Biochemistry 57, 2478-2487 (2018).

3. Kato, M. et al. Cell-free formation of RNA granules: Low complexity sequence domains form dynamic fibers within hydrogels. Cell 149, 753-767 (2012).

4. Lin, Y., Protter, D. S. W., Rosen, M. K. \& Parker, R. Formation and maturation of phase-separated liquid droplets by RNA-binding proteins. Mol. Cell 60, 208-219 (2015). 
5. King, O. D., Gitler, A. D. \& Shorter, J. The tip of the iceberg: RNA-binding proteins with prion-like domains in neurodegenerative disease. Brain Res. 1462, 61-80 (2012).

6. Kim, H. J. et al. Mutations in prion-like domains in hnRNPA2B1 and hnRNPA1 cause multisystem proteinopathy and ALS. Nature 495, 467-473 (2013).

7. Molliex, A. et al. Phase separation by low complexity domains promotes stress granule assembly and drives pathological fibrillization. Cell 163, 123-133 (2015).

8. Schwartz, J. C. et al. FUS binds the CTD of RNA polymerase II and regulates its phosphorylation at Ser2. Genes Dev. 26, 2690-2695 (2012).

9. Kwon, I. et al. Phosphorylation-regulated binding of RNA polymerase II to fibrous polymers of low-complexity domains. Cell 155, 1049-1060 (2013).

10. Lagier-Tourenne, C. et al. Divergent roles of ALS-linked proteins FUS/TLS and TDP-43 intersect in processing long pre-mRNAs. Nat. Neurosci. 15, 1488-1497 (2012).

11. Ling, S. C., Polymenidou, M. \& Cleveland, D. W. Converging mechanisms in ALS and FTD: disrupted RNA and protein homeostasis. Neuron 79, 416-438 (2013).

12. Deng, Q. D. et al. FUS is phosphorylated by DNA-PK and accumulates in the cytoplasm after DNA damage. J. Neurosci. 34, 7802-7813 (2014).

13. Rhoads, S. N. et al. The prionlike domain of FUS is multiphosphorylated following DNA damage without altering nuclear localization. Mol. Biol. Cell 29, 1786-1797 (2018).

14. Sun, Z. H. et al. Molecular determinants and genetic modifiers of aggregation and toxicity for the ALS disease protein FUS/TLS. PLoS Biol. 9, e1000614 (2011).

15. Patel, A. et al. A liquid-to-solid phase transition of the ALS protein FUS accelerated by disease mutation. Cell 162, 1066-1077 (2015).

16. Fujii, R. et al. The RNA binding protein TLS is translocated to dendritic spines by mGluR5 activation and regulates spine morphology. Curr. Biol. 15, 587-593 (2005).

17. Burke, K. A., Janke, A. M., Rhine, C. L. \& Fawzi, N. L. Residue-by-residue view of in vitro FUS granules that bind the C-terminal domain of RNA polymerase II. Mol. Cell 60, 231-241 (2015).

18. Zinszner, H., Sok, J., Immanuel, D., Yin, Y. \& Ron, D. T. L. S. (FUS) binds RNA in vivo and engages in nucleo-cytoplasmic shuttling. J. Cell Sci. 110, 1741-1750 (1997).

19. Schwartz, J. C., Wang, X. Y., Podell, E. R. \& Cech, T. R. RNA seeds higherorder assembly of FUS protein. Cell Rep. 5, 918-925 (2013).

20. Yang, L. Q., Gal, J., Chen, J. \& Zhu, H. N. Self-assembled, F. U. S. binds active chromatin and regulates gene transcription. Proc. Natl Acad. Sci. USA 111, 17809-17814 (2014).

21. Murray, D. T. et al. Structure of FUS protein fibrils and its relevance to selfassembly and phase separation of low-complexity domains. Cell 171, 615-627 (2017).

22. Ding, X. F. et al. Amyloid-forming segment induces aggregation of FUS-LC domain from phase separation modulated by site-specific phosphorylation. $J$. Mol. Biol. 432, 467-483 (2020)

23. Murakami, T. et al. ALS/FTD mutation-induced phase transition of FUS liquid droplets and reversible hydrogels into irreversible hydrogels impairs RNP granule function. Neuron 88, 678-690 (2015).

24. Matsumoto, T. et al. Self-assembly of FUS through its low-complexity domain contributes to neurodegeneration. Hum. Mol. Genet. 27, 1353-1365 (2018).

25. Kwiatkowski, T. J. et al. Mutations in the FUS/TLS gene on chromosome 16 cause familial amyotrophic lateral sclerosis. Science 323, 1205-1208 (2009).

26. Vance, C. et al. Mutations in FUS, an RNA processing protein, cause familial amyotrophic lateral sclerosis type 6. Science 323, 1208-1211 (2009).

27. Deng, H., Gao, K. \& Jankovic, J. The role of FUS gene variants in neurodegenerative diseases. Nat. Rev. Neurol. 10, 337-348 (2014).

28. Niaki, A. G. et al. Loss of dynamic RNA interaction and aberrant phase separation induced by two distinct types of ALS/FTD-linked FUS mutations. Mol. Cell 77, 82-94 (2020).

29. Scekic-Zahirovic, J. et al. Toxic gain of function from mutant FUS protein is crucial to trigger cell autonomous motor neuron loss. EMBO J. 35, 1077-1097 (2016).

30. Murray, D. T. \& Tycko, R. Side chain hydrogen-bonding interactions within amyloid-like fibrils formed by the low-complexity domain of FUS: Evidence from solid state nuclear magnetic resonance spectroscopy. Biochemistry 59, 364-378 (2020).

31. Murray, D. T. et al. Molecular structure of FUS low sequence complexity domain protein fibrils. Protein Data Bank, https://doi.org/10.2210/pdb5W3N/ pdb (2017)

32. Tycko, R., Lee, M., Ghosh, U., Thurber, K. R. \& Kato, M. Molecular structure of the core of amyloid-like fibrils formed by residues 111-214 of FUS. Protein Data Bank. https://doi.org/10.2210/pdb6XFM/pdb (2020).

33. Chen, B., Thurber, K. R., Shewmaker, F., Wickner, R. B. \& Tycko, R. Measurement of amyloid fibril mass-per-length by tilted-beam transmission electron microscopy. Proc. Natl Acad. Sci. USA 106, 14339-14344 (2009)
34. He, S. D. \& Scheres, S. H. W. Helical reconstruction in Relion. J. Struct. Biol. 198, 163-176 (2017).

35. Scheres, S. H. W. Relion: implementation of a Bayesian approach to cryo-EM structure determination. J. Struct. Biol. 180, 519-530 (2012).

36. Ghosh, U., Thurber, K. R., Yau, W. M. \& Tycko, R. Molecular structure of a prevalent amyloid- $\beta$ fibril polymorph from Alzheimer's disease brain tissue. Preprint at https://www.biorxiv.org/content/ https://doi.org/10.1101/ 2020.03.06.981381v1 (2020).

37. Emsley, P., Lohkamp, B., Scott, W. G. \& Cowtan, K. Features and development of Coot. Acta Crystallogr. D. Biol. Crystallogr. 66, 486-501 (2010).

38. Schwieters, C. D., Kuszewski, J. J. \& Clore, G. M. Using Xplor-NIH for NMR molecular structure determination. Prog. Nucl. Magn. Reson. Spectrosc. 48, 47-62 (2006)

39. Sawaya, M. R. et al. Atomic structures of amyloid cross- $\beta$ spines reveal varied steric zippers. Nature 447, 453-457 (2007).

40. Perutz, M. F., Johnson, T., Suzuki, M. \& Finch, J. T. Glutamine repeats as polar zippers: Their possible role in inherited neurodegenerative diseases. Proc. Natl Acad. Sci. USA 91, 5355-5358 (1994).

41. McGaughey, G. B., Gagne, M. \& Rappe, A. K. Pi-stacking interactions: Alive and well in proteins. J. Biol. Chem. 273, 15458-15463 (1998).

42. Gazit, E. A possible role for pi-stacking in the self-assembly of amyloid fibrils. Faseb J. 16, 77-83 (2002).

43. Morris, G. A. \& Freeman, R. Enhancement of nuclear magnetic resonance signals by polarization transfer. J. Am. Chem. Soc. 101, 760-762 (1979).

44. Siemer, A. B. et al. Observation of highly flexible residues in amyloid fibrils of the HET-s prion. J. Am. Chem. Soc. 128, 13224-13228 (2006).

45. Helmus, J. J., Surewicz, K., Surewicz, W. K. \& Jaroniec, C. P. Conformational flexibility of Y145Stop human prion protein amyloid fibrils probed by solidstate nuclear magnetic resonance spectroscopy. J. Am. Chem. Soc. 132, 2393-2403 (2010)

46. Wishart, D. S., Bigam, C. G., Holm, A., Hodges, R. S. \& Sykes, B. D. ${ }^{1}$ H. ${ }^{13}$ C and ${ }^{15} \mathrm{~N}$ random coil NMR chemical shifts of the common amino acids .1. Investigations of nearest-neighbor effects. J. Biomol. NMR 5, 67-81 (1995)

47. Phillips, J. C. et al. Scalable molecular dynamics with NAMD. J. Comput. Chem. 26, 1781-1802 (2005).

48. Hughes, M. P. et al. Atomic structures of low-complexity protein segments reveal kinked $\beta$ sheets that assemble networks. Science 359, 698-701 (2018).

49. Luo, F. et al. Atomic structures of FUS LC domain segments reveal bases for reversible amyloid fibril formation. Nat. Struct. Mol. Biol. 25, 341-346 (2018).

50. Schutz, A. K. et al. Atomic-resolution three-dimensional structure of amyloid $\beta$ fibrils bearing the Osaka mutation. Angew. Chem. Int. Ed. 54, 331-335 (2015).

51. Tuttle, M. D. et al. structure of a pathogenic fibril of full-length human asynuclein. Nat. Struct. Mol. Biol. 23, 409-415 (2016).

52. Hervas, R. et al. Cryo-EM structure of a neuronal functional amyloid implicated in memory persistence in Drosophila. Science 367, 1230-1234 (2020).

53. Falcon, B. et al. Structures of filaments from Pick's disease reveal a novel tau protein fold. Nature 561, 137-140 (2018).

54. Gremer, L. et al. Fibril structure of amyloid- $\beta(1-42)$ by cryo-electron microscopy. Science 358, 116-119 (2017).

55. Qiang, W., Kelley, K. \& Tycko, R. Polymorph-specific kinetics and thermodynamics of $\beta$-amyloid fibril growth. J. Am. Chem. Soc. 135 , 6860-6871 (2013)

56. Bernado, P. \& Svergun, D. I. Structural analysis of intrinsically disordered proteins by small-angle x-ray scattering. Mol. Biosyst. 8, 151-167 (2012).

57. Zhou, H. X. \& Dill, K. A. Stabilization of proteins in confined spaces. Biochemistry 40, 11289-11293 (2001).

58. Wiltzius, J. J. W. et al. Atomic structure of the cross- $\beta$ spine of islet amyloid polypeptide (amylin). Protein Sci. 17, 1467-1474 (2008).

59. Guenther, E. L. et al. Atomic structures of TDP-43 LCD segments and insights into reversible or pathogenic aggregation. Nat. Struct. Mol. Biol. 25, 463-471 (2018).

60. Cao, Q., Boyer, D. R., Sawaya, M. R., Ge, P. \& Eisenberg, D. S. Cryo-EM structures of four polymorphic TPD-43 amyloid cores. Nat. Struct. Mol. Biol. 26, 619-627 (2019).

61. Lu, J. X. et al. Molecular structure of $\beta$-amyloid fibrils in Alzheimer's disease brain tissue. Cell 154, 1257-1268 (2013).

62. Colvin, M. T. et al. Atomic resolution structure of monomorphic A $\beta(42)$ amyloid fibrils. J. Am. Chem. Soc. 138, 9663-9674 (2016).

63. Ni, X. D., McGlinchey, R. P., Jiang, J. S. \& Lee, J. C. Structural insights into asynuclein fibril polymorphism: effects of Parkinson's disease-related Cterminal truncations. J. Mol. Biol. 431, 3913-3919 (2019).

64. Van Melckebeke, H. et al. Atomic-resolution three-dimensional structure of HET-s(218-289) amyloid fibrils by solid-state NMR spectroscopy. J. Am. Chem. Soc. 132, 13765-13775 (2010).

65. El Mammeri, N. et al. Molecular architecture of bacterial amyloids in Bacillus biofilms. Faseb J. 33, 12146-12163 (2019). 
66. Nagaraj, M. et al. Predicted loop regions promote aggregation: a study of amyloidogenic domains in the functional amyloid FAPC. J. Mol. Biol. 432, 2232-2252 (2020).

67. Petkova, A. T., Yau, W. M. \& Tycko, R. Experimental constraints on quaternary structure in Alzheimer's $\beta$-amyloid fibrils. Biochemistry 45, 498-512 (2006).

68. Glynn, C. et al. Cryo-EM structure of a human prion fibril with a hydrophobic, protease-resistant core. Nat. Struct. Mol. Biol. 27, 417-423 (2020).

69. Paravastu, A. K., Leapman, R. D., Yau, W. M. \& Tycko, R. Molecular structural basis for polymorphism in Alzheimer's $\beta$-amyloid fibrils. Proc. Nat Acad. Sci. USA 105, 18349-18354 (2008).

70. Chiti, F. et al. Designing conditions for in vitro formation of amyloid protofilaments and fibrils. Proc. Natl Acad. Sci. USA 96, 3590-3594 (1999).

71. Takegoshi, K., Nakamura, S. \& Terao, T. ${ }^{13} \mathrm{C}-{ }^{1} \mathrm{H}$ dipolar-assisted rotational resonance in magic-angle spinning NMR. Chem. Phys. Lett. 344, 631-637 (2001).

72. Bennett, A. E., Rienstra, C. M., Auger, M., Lakshmi, K. V. \& Griffin, R. G. Heteronuclear decoupling in rotating solids. J. Chem. Phys. 103, 6951-6958 (1995).

73. Tycko, R., Pines, A. \& Guckenheimer, J. Fixed-point theory of iterative excitation schemes in NMR. J. Chem. Phys. 83, 2775-2802 (1985).

74. Delaglio, F. et al. Nmrpipe: A multidimensional spectral processing system based on Unix pipes. J. Biomol. NMR 6, 277-293 (1995).

75. Pettersen, E. F. et al. UCSF Chimera: a visualization system for exploratory research and analysis. J. Comput. Chem. 25, 1605-1612 (2004).

76. Best, R. B. et al. Optimization of the additive CHARMM all-atom protein force field targeting improved sampling of the backbone $\phi, \psi$ and sidechain $\chi_{1}$ and $\chi_{2}$ dihedral angles. J. Chem. Theory Comput. 8, 3257-3273 (2012).

77. Humphrey, W., Dalke, A. \& Schulten, K. VMD: visual molecular dynamics. J. Mol. Graph. 14, 33-38 (1996).

\section{Acknowledgements}

This work was supported by the Intramural Research Program of the National Institute of Diabetes and Digestive and Kidney Diseases, National Institutes of Health. Cryo-EM data were obtained at the NIH Multi-Institute Cryo-EM Facility (MICEF). Calculations of density maps and molecular models were performed on the NIH High Performance Computing Biowulf cluster.

\section{Author contributions}

M.L. and M.K. prepared protein and fibril samples. M.L., U.G., and K.R.T. acquired and analyzed cryo-EM data. M.L., M.K., and R.T. acquired and analyzed NMR data and performed other measurements. M.L. and R.T. wrote the manuscript.

\section{Competing interests}

The authors have no competing interests.

\section{Additional information}

Supplementary information is available for this paper at https://doi.org/10.1038/s41467020-19512-3.

Correspondence and requests for materials should be addressed to R.T.

Peer review information Nature Communications thanks Ümit Akbey, Haifeng Chen, and the other, anonymous, reviewer(s) for their contribution to the peer review of this work. Peer reviewer reports are available.

Reprints and permission information is available at http://www.nature.com/reprints

Publisher's note Springer Nature remains neutral with regard to jurisdictional claims in published maps and institutional affiliations.

(c) (i) Open Access This article is licensed under a Creative Commons Attribution 4.0 International License, which permits use, sharing, adaptation, distribution and reproduction in any medium or format, as long as you give appropriate credit to the original author(s) and the source, provide a link to the Creative Commons license, and indicate if changes were made. The images or other third party material in this article are included in the article's Creative Commons license, unless indicated otherwise in a credit line to the material. If material is not included in the article's Creative Commons license and your intended use is not permitted by statutory regulation or exceeds the permitted use, you will need to obtain permission directly from the copyright holder. To view a copy of this license, visit http://creativecommons.org/ licenses/by/4.0/.

This is a U.S. government work and not under copyright protection in the U.S.; foreign copyright protection may apply 2020 